\title{
Modelling of JET hybrid plasmas with emphasis on performance of combined ICRF and NBI heating
}

D. Gallart ${ }^{1}$, M.J. Mantsinen ${ }^{1,2}$, C. Challis ${ }^{3}$, D. Frigione ${ }^{4}$, J. Graves $^{5}$, E. Belonohy ${ }^{6}$, F. Casson ${ }^{3}$, A. Czarnecka ${ }^{7}$, J. Eriksson ${ }^{8}$, J. Garcia ${ }^{9}$, M. Goniche ${ }^{9}$, C. Hellesen ${ }^{8}$, J. Hobirk ${ }^{6}$, P. Jaquet ${ }^{3}$, E. Joffrin ${ }^{9}$, N. Krawczyk ${ }^{7}$, D. King ${ }^{3}$, M. Lennholm ${ }^{10}$, E. Lerche $^{11}$, E. Pawelec ${ }^{12}$, X. Sáez ${ }^{1}$, M. Sertoli ${ }^{6}$, G. Sips ${ }^{6}$, E. Solano $^{13}$, M. Tsalas ${ }^{14}$, P. Vallejos ${ }^{8}$, M. Valisa ${ }^{15}$ and JET Contributors

${ }^{1}$ Barcelona Supercomputing Center (BSC)

${ }^{2}$ ICREA, Barcelona, Spain

${ }^{3}$ CCFE, Culham Science Centre, Abingdon, Oxon, OX14 3DB, UK

${ }^{4}$ Associazione EURATOM-ENEA, C.R.E. Frascati, Italy

${ }^{5}$ Centre de Recherches en Physique des Plasmas, EPFL, 1015, Lausanne, Switzerland

${ }^{6}$ Max-Planck-Institut fur Plasmaphysik, Garching, Germany

${ }^{7}$ Institute of Plasma Physics and Laser Microfusion, Association EURATOM/IPPLM, Poland

${ }^{8}$ Uppsala University, Euratom/VR Fusion Assoc., Uppsala, Sweden

${ }^{9} \mathrm{CEA}$, Centre d'Etudes Nucleaires de Cadarache, France

${ }^{10}$ European Comission, B-1049 Brussels, Belgium

${ }^{11}$ Laboratory for Plasma Physics, LPP-ERM/KMS, Brussels, Belgium

${ }^{12}$ Institute of Physics, Opole University, ul. Oleska 48, Opole 45-052, Poland

${ }^{13}$ Association EURATOM-CIEMAT para Fusion, CIEMAT, Madrid, Spain

${ }^{14}$ FOM institute DIFFER, Association EURATOM-FOM, PO Box 120, Nieuwegein, Netherlands

${ }^{15}$ Consorzio RFX, Associazione Euratom-ENEA sulla Fusione, Padova 35137, Italy

E-mail: daniel.gallart@bsc.es

\begin{abstract}
During the 2015-2016 JET campaigns many efforts have been devoted to the exploration of high-performance plasma scenarios envisaged for DT operation in JET. In this paper we review various key recent hybrid discharges and model the combined ICRF+NBI heating. These deuterium discharges with deuterium beams had the ICRF antenna frequency tuned to match the cyclotron frequency of minority $\mathrm{H}$ at the centre of the tokamak coinciding with the second harmonic cyclotron resonance of D. The modelling takes into account the synergy between ICRF and NBI heating through the second harmonic cyclotron resonance of $\mathrm{D}$ beam ions which allows us to assess its impact on the neutron rate $R_{N T}$. We evaluate the influence of the resonance position and $\mathrm{H}$ concentration which were varied in different discharges in order to test their role in the heating performance. It was found that discharges with a resonance beyond $\sim 0.15 \mathrm{~m}$ from the magnetic axis $R_{0}$ suffered from impurity accumulation in these plasma conditions. According to our modelling, the ICRF enhancement of $R_{N T}$ increases with the ICRF power absorbed by deuterons as the $\mathrm{H}$ concentration decreases. We find that in the recent hybrid discharges this ICRF enhancement varied
\end{abstract}


due to a variation of $\mathrm{H}$ concentration and was in the range of $10-25 \%$. The modelling of a recent record high-performance hybrid discharge shows that ICRF fusion yield enhancement of $\sim 30 \%$ and $\sim 15 \%$ can be achieved in the ramp-up phase and during the main heating phase, respectively. We extrapolate the results to DT and find that the best performing hybrid discharges correspond to an equivalent fusion power of $\sim 7.0 \mathrm{MW}$ in DT. Finally, an optimization analysis of the bulk ion heating for the DT scenario reveals around $15-20 \%$ larger bulk ion heating for the ${ }^{3} \mathrm{He}$ minority scenario as compared to the $\mathrm{H}$ minority scenario. 


\section{Introduction}

Among the envisaged auxiliary heating systems for ITER, ion cyclotron resonance frequency (ICRF) heating has proved to be an efficient mechanism to bring plasmas at high temperatures in present-day tokamaks. ICRF heating demonstrated its capabilities for heating of reactor-like plasmas in DT scenarios relevant for ITER in TFTR $[1,2]$ and during the 1997 DT campaign in JET [3-5]. Several studies have been conducted for the initial non-activated phase of ITER, where the reference ICRF heating scenarios rely on minority species such as 3 -helium $\left({ }^{3} \mathrm{He}\right)$ or deuterium (D) in hydrogen $(\mathrm{H})$ majority plasmas $[6,7]$. Moreover, ICRF has many applications beyond heating due to its interaction with the plasma [8], bringing the flexibility for a wide range of different experiments such as the mimicking of fusion-born alphas [9] or the production of super energetic particles with the novel three-ion scheme [10]. ICRF heating also plays an important role in achieving high-performance discharges via optimization of bulk ion heating [11-13], fusion enhancement [14,15] and core impurity control [16-18].

During the 2015-2016 JET campaigns with the ITER-like-wall (ILW) many efforts have been devoted to the exploration of high-performance plasma scenarios envisaged for ITER operation [19]. The inductive (baseline) scenario [20] and the hybrid scenario [21] have achieved major improvements during these campaigns surpassing the previous ILW fusion record of $2.3 \cdot 10^{16}$ neutrons/s, thus showing good progress towards demonstrating the fusion rate goal for DT (the objective for DT-ready plasma is $6 \cdot 10^{16} \mathrm{~s}^{-1}$ for $5 \mathrm{~s}[22]$ ). The hybrid scenario reached with $33 \mathrm{MW}$ of combined ICRF and NBI power a record neutron rate $R_{N T}$ of $2.9 \cdot 10^{16} \mathrm{~s}^{-1}$. The hybrid scenario is an advanced regime expected to be applied in ITER. It is characterized by a low plasma current $I_{p}$ which allows operation at a high normalised beta $\beta_{N}=\langle\beta\rangle a B_{T} / I_{p}$ and a $q$ profile $(q(0) \geq 1)$ that prevents sawtoothing $\mathrm{m}=1, \mathrm{n}=1(1 / 1)$ MHD activity in the core from triggering large NTMs [21]. In preparation for the next DT campaign at JET with the ILW planned for 2020, the analysis of this scenario's heating performance is of great relevance as it gives insight on the strategy to follow in order to obtain the objective of a fusion reaction rate of $6 \cdot 10^{16} \mathrm{~s}^{-1}$ for $5 \mathrm{~s}[22]$.

This paper studies the heating performance of the recent hybrid discharges where we model and assess the performance of ICRF +NBI heating and the fusion enhancement through ICRF heating. The aim is to predict the fusion performance of the best discharges with a DT plasma giving an estimation of their associated fusion power. Hence, this paper provides the reader with a description of the ICRF and NBI heating modelling and the impact of these heating mechanisms on the fusion yield of the recent hybrid discharges at JET. In order to do so, we evaluate the performance of $\mathrm{H}$ minority heating in the presence of D beam ions using the ICRF modelling code PION [23] coupled [11] to the beam deposition code PENCIL [24]. Our modelling also takes into account the synergy between ICRF and NBI heating through the second harmonic cyclotron resonance of $\mathrm{D}$ beam ions, which allows us to assess its impact on the fusion performance. The hybrid discharges analysed in this paper were composed of minority 
$\mathrm{H}$ and a majority of $\mathrm{D}$ together with a low concentration of certain impurities such as beryllium (Be) or carbon (C). The antenna was tuned to the $\mathrm{H}$ fundamental resonance which coincides with the $2^{\text {nd }} \mathrm{D}$ harmonic resonance $\left(\omega=\omega_{H}=2 \omega_{D}\right)$ and in most cases it was a central resonance, $s \approx 0.05-0.1$ and ranging to $s \approx 0.2$ for discharges with an off-axis resonance, where $s$ is the square-root of the normalised poloidal flux. The analysis shows that for optimal plasma conditions with high plasma temperature, low $\mathrm{H}$ concentration and the presence of $\mathrm{D}$ beams, most of the ICRF power is coupled to D which has beneficial effects to the fusion yield.

Given the relevance and interest shown in the forthcoming 2018-2020 JET campaigns in the performance of different ICRF heating schemes in order to boost heating and fusion yield, we have prepared, in addition to the modelling of the experimental scenarios with $\mathrm{H}$ minority, a comparison in section 8.2 of ${ }^{3} \mathrm{He}$ and $\mathrm{H}$ minority in a DT plasma where we assess the bulk ion heating efficiency under a scan of relevant plasma parameters. For these cases, the antenna frequency was tuned to the ${ }^{3} \mathrm{He}$ fundamental resonance which coincides with the $2^{\text {nd }}$ tritium $(\mathrm{T})$ harmonic resonance ( $\left.\omega=\omega_{3}{ }_{H e}=2 \omega_{T}\right)$. Central heating with a resonance at $s=0.01-0.05$ was considered.

We model a number of key discharges that were designed in order to evaluate key ICRF aspects. Therefore, in order to assess the best strategy to follow in terms of ICRF heating we have organised our modelling analysis in the following way: (1) the avoidance of impurity accumulation with ICRF waves and its dependence on the ICRF resonance position, (2) the impact of $\mathrm{H}$ concentration on the ICRF damping mechanisms, i.e, the fundamental $\mathrm{H}$ resonance, the $2^{\text {nd }} \mathrm{D}$ harmonic resonance and the direct electron damping, (3) the analysis of combined ICRF and NBI in high-performance hybrid discharges and their associated ICRF fusion performance enhancement. And (4) the prediction for a record discharge to a 50:50 DT fuel mixture including the analysis of the optimal conditions to maximize bulk ion heating.

In order to validate our modelling for JET discharges we can not rely on direct measurements of ICRF local power density absorption as they are not available. However, we have calculated ICRF-related quantities such as the neutron production and the enhancement by ICRF of the fusion yield and then compared with the experimental data to show that our modelling is consistent with the experiments.

Including this introduction our paper is organised in 8 sections comprising the theoretical background of the analysis (section 2), the numerical methods used (section 3 ), the general parameters of the hybrid scenario studied (section 4), the discussion of the modelling results (sections 5-8) and finally the conclusions (section 9). This paper is the continuation and the extension of a series of papers devoted to the ICRF modelling of hybrid discharges Refs. [25-28].

\section{Theoretical Background}

Here we discuss the ICRF physics involved in the analysis of the modelling results, especially those that have direct impact on the fusion performance: ICRF power 
partition, power partitioning between ions and electrons and mechanisms to influence impurity transport by ICRF waves.

\subsection{Power partition}

Three damping mechanisms compete in the discharges studied in this paper, i.e., the ion cyclotron resonance of $\mathrm{H}$ and $\mathrm{D}$ through fundamental hydrogen resonance $\left(\omega=\omega_{H}\right)$ and $2^{\text {nd }} \mathrm{D}$ harmonic resonance $\left(\omega=2 \omega_{D}\right)$, direct electron damping via electron Landau Damping (ELD) and transit time magnetic pumping (TTMP). Direct electron damping may play an important role in those discharges with high electron densities and temperatures and when the resonance position is located at the high-field side (HFS). For the plasma temperatures and densities of this hybrid high-performing discharges direct electron damping typically damps $\sim 10 \%$ of the wave energy. Fundamental $\mathrm{H}$ damping is important during the ramp-up, when the plasma is still cold and in the absence of $\mathrm{D}$ beams. The $2^{\text {nd }} \mathrm{D}$ harmonic depends strongly on the velocity distribution of $\mathrm{D}$, becoming more relevant as an energetic $\mathrm{D}$ tail develops, i.e. with the $\mathrm{D}$ beams. The local power partition between $\mathrm{D}$ harmonic and $\mathrm{H}$ fundamental scales to lowest order as $[11,23]$ :

$$
\frac{p_{2}}{p_{1}}=c_{21} \frac{k_{\perp}^{2} w_{2}}{n_{1} m_{1} \omega_{c 2}^{2}} .
$$

Here, the subscripts 1 and 2 refer to the resonant species with a fundamental and a $2^{\text {nd }}$ harmonic resonance, respectively, $p_{1}$ and $p_{2}$ are the local power densities absorbed by $\mathrm{H}$ and $\mathrm{D}$, respectively, $k_{\perp}$ is the perpendicular wavenumber, $w_{2}$ is the energy density of deuterium, $n_{1}$ and $m_{1}$ are the hydrogen density and mass, respectively, $\omega_{c 2}$ is the cyclotron frequency of D and $c_{21}$ is a constant. The analysis of JET high-performance discharges with PION shows that $c_{21} \approx 0.2-0.3$.

In the experiments we lowered the $\mathrm{H}$ concentration so the $\mathrm{D}$ power absorption increased, as can be seen in (1). Another important factor is the D beam injection which increases substantially the D energy density and consequently the D power absorption. These two joint effects contributed to achieving the highest fusion yield that has been reached in the hybrid discharges.

For the DT prediction we have considered two ICRF scenarios, ${ }^{3} \mathrm{He}$ minority and $\mathrm{H}$ minority. ${ }^{3} \mathrm{He}$ minority resulted in a strong damping performance. ${ }^{3} \mathrm{He}$ absorbs most of the wave energy during all the stages of the discharge, in contrast with the $\mathrm{H}$ minority damping performance which typically becomes weaker as D beams are injected. For this scenario the equation (1) is still valid, taking into account that subscripts 1 and 2 refer to ${ }^{3} \mathrm{He}$ and $\mathrm{T}$, respectively. The computed value of constant $c_{21}$ based on PION results is $c_{21} \approx 0.01-0.02$.

The $3^{\text {rd }}$ harmonic resonance must be taken into account in the DT prediction with $\mathrm{D}$ and $\mathrm{T}$ beams when the minority species considered is $\mathrm{H}$. For $\mathrm{H}$ minority in DT plasma, the damping performance from resonant $\mathrm{H}$ and $\mathrm{D}$ ions is similar to that from a pure $\mathrm{D}$ plasma. In fact, the $3^{\text {rd }} \mathrm{T}$ harmonic resonance does not play a relevant role as it absorbs 
a small fraction of the wave energy, which is negligible compared to that absorbed by $\mathrm{H}$ or D. In order to understand this behavior, we have obtained an equation that describes the local power partition for the $3^{\text {rd }}$ harmonic using the same procedure to compute (1). The local power partition between the $3^{\text {rd }}$ harmonic and fundamental resonant ions scales to lowest order as,

$$
\frac{p_{3}}{p_{1}}=c_{31} \frac{m_{3} k_{\perp}^{4}}{m_{1} n_{1} \omega_{c 3}^{4}} \int v_{3}^{4} f_{3} d v .
$$

The main difference from (1) comes from the presence of the 4th moment of the distribution function which needs to be computed numerically and the different exponents in the wave number and the cyclotron frequency. However, by assessing the order of magnitude of the terms $k_{\perp}^{2} w_{2} / \omega_{c 2}^{2}$ and $k_{\perp}^{4} m_{3} \int v_{3}^{4} f_{3} d v / \omega_{c 3}^{4}$ for (1) and (2), respectively, for typical values of the scenarios studied we find that there are approximately two orders of magnitude of difference between them, being higher the term in (1). This theoretical approximation explains the weak damping strength observed for the $3^{\text {rd }} \mathrm{T}$ harmonic resonance in the simulations performed with PION.

\subsection{Bulk ion heating}

Major bulk ion heating is necessary to increase the number of thermal fusion reactions. In order to obtain major bulk ion heating the fast ions average energy should not surpass a threshold energy, the so called critical energy. However, ions become substantially more energetic than thermal ions in the resonance location where the resonant waveparticle interaction takes place. In the process of thermalisation, energetic ions will slow-down by colliding with background ions and electrons, this effect increases the thermal ion and electron temperature, respectively. The energy threshold at which thermal ions and electrons are equally heated is the critical energy [35],

$$
E_{\text {crit }}=14.8 A T_{e}\left[\sum_{j} \frac{n_{j} Z_{j}^{2}}{n_{e} A_{j}}\right]^{\frac{2}{3}} .
$$

Here $A$ is the atomic mass of the resonant ion species, $T_{e}$ is the electron temperature, $n_{j}$ and $n_{e}$ are the densities of the $j$-ith ion species and electrons, respectively, $Z_{j}$ and $A_{j}$ are the atomic number and atomic mass of the $j$-ith ion species, respectively.

The velocity distribution of the resonant ions plays a crucial role in the way the collisional power is transferred. The average fast ion energy must be kept below the critical energy for dominant bulk ion heating. An energetic ion of energy E transfers its energy following $\dot{W}_{i} / \dot{W}_{e}=\left(E_{\text {crit }} / E\right)^{3 / 2}$ where $\dot{W}_{i}$ and $\dot{W}_{e}$ are the rate of energy transfer to thermal ions and electrons, respectively, while $\mathrm{E}$ is the energy of the nonthermal ion [35]. This is the instantaneous ratio of energy transfer from an ion to thermal ions and electrons, for a complete slowing-down one needs to integrate over all the energy states of the ion until thermalisation is reached. Here, we show the ratio of the total energy given up by a single ion of energy $E$ to thermal ions after a complete thermalisation as done by Stix in [35] and as an extension to this work we 
have integrated this ratio over a Maxwellian, which gives an approximation of the energy transferred from fast ions to thermal ions in a simplified case. However, ICRF heating through $2^{\text {nd }} \mathrm{D}$ harmonic resonance drives a strong tail in the $\mathrm{D}$ velocity distribution function which is non-Maxwellian and, therefore, figure 1 must be understood as a rough estimation. It has been computed as $\int_{0}^{E} G(E) f(E) d E$, where $G(E)$ is the average fraction of the energy transferred from fast ions to thermal ions as defined in [35] and $f(E)$ is a maxwellian distribution for fast ions (figure 1). In the best performing hybrid
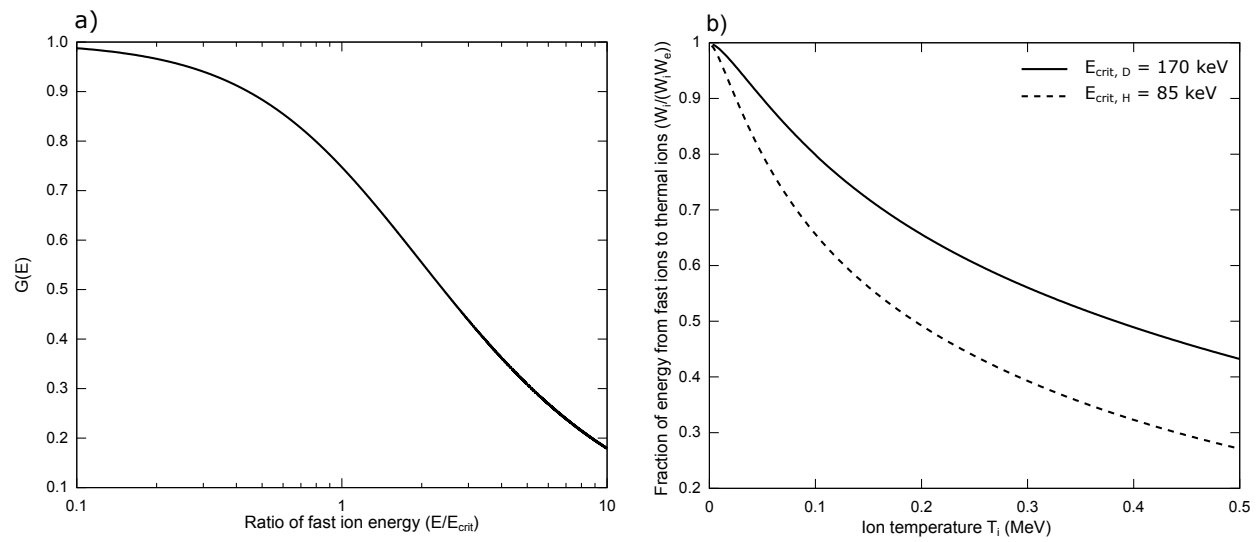

Figure 1: a) Ratio of energy $G(E)$ given up by a fast ion to thermal ions over a complete thermalisation as in [35], b) $G(E)$ averaged over a maxwellian $f(E)$ for different thermodynamic ion temperatures $T_{i}$ and fixed critical energies for $D$ and $H$, $E_{\text {crit }, D}=170$ and $E_{\text {crit }, H}=85 \mathrm{keV}$, respectively.

experiments with $\mathrm{H}$ minority and $\mathrm{D}$ majority the electron temperature was about 10 $\mathrm{keV}$ and the plasma density of $3-6 \cdot 10^{20} \mathrm{~m}^{-3}$. In these conditions $E_{\text {crit }, H} \sim 75-110 \mathrm{keV}$ for $\mathrm{H}$ and $E_{\text {crit,D }} \sim 150-220 \mathrm{keV}$ for $\mathrm{D}$ in the plasma centre according to (3). Resonant $\mathrm{H}$ ions have an average fast ion energy around $160 \mathrm{keV}$ during the main heating phase for the experiments presented in this paper. From the simplified calculation shown in figure $1 \mathrm{~b}$ ) one expects to have dominant bulk ion heating from fast $\mathrm{H}$ ions. This is not the case during the ramp-up where resonant $\mathrm{H}$ ions reach an average energy of 500 keV and as will be shown, collisions with electrons are dominant. Taking into account that $\mathrm{D}$ beams are injected at an energy around $110 \mathrm{keV}$ dominant bulk ion heating is expected (figure $1 \mathrm{~b}$ )), the addition of ICRF heating can bring a fraction of D ions to energy levels closer or even above the D critical energy. In a pure D plasma, where the $\mathrm{DD}$ fusion cross section peaks at the $\mathrm{MeV}$ range, the acceleration of $\mathrm{D}$ ions by ICRF waves substantially enhances the fusion yield as will be shown in the paper. However, in a DT plasma, the fusion cross section peaks around $120 \mathrm{keV}$ for fast D. Therefore, the acceleration of D ions by ICRF enhancement over pure NBI might lead the beams to energies above the injection energy which might decrease the fusion neutron rate and bulk ion heating if the average energy reached is beyond $120 \mathrm{keV}$ or $E_{\text {crit }}$, respectively. 


\subsection{On mechanisms to influence $W$ accumulation with ICRF waves}

Tungsten $(\mathrm{W})$ in the divertor, Be limiters and exposed inconnel in main chamber are currently the plasma wall facing components of the installed ILW at JET. Tungsten possesses a high temperature tolerance and low erosion rate which make it a strong candidate for the future ITER reactor. However, dilution by any high-Z impurities need to be controled and minimised below a concentration of $10^{-4}$ in a fusion plasma reactor in order to achieve high-performance discharges.

When poloidal asymmetries are not considered and following the discussion in $[17,29] \mathrm{W}$ flux can be described as:

$$
\Gamma_{W} \sim n_{i} T_{i} \nu_{i W} Z_{W}\left(\frac{R}{L_{n_{i}}}-0.5 \frac{R}{L_{T_{i}}}\right) .
$$

Here, $n_{i}$ and $T_{i}$ are the ion density and temperature, respectively, $\nu_{i W} \sim T_{i}^{-3 / 2}$ is the background ion-tungsten collision frequency, $R$ is the major radius and $L_{n_{i}}$ and $L_{T_{i}}$ are the inverse of the logarithmic gradient of ion density and temperature, respectively. For ion temperature gradient terms larger than the ion density gradient term, central W accumulation is avoided as the flux convection becomes outward.

Recent studies $[17,18,29,33]$ have proved ICRF heating as an efficient mechanism to avoid central impurity accumulation, mainly through avoidance of poloidal asymmetries and enhancement of temperature screening. In the presence of ICRF heating, temperature screening in (4) is effectively increased by the fast minority temperature screening $\sim n_{f} T_{f}^{-1 / 2} R / L_{T_{f}}$. Furthermore, fast minority ions tend to become trapped in banana orbits that are localised at the low-field side (LFS) and create a poloidal varying potential that push $\mathrm{W}$ to the high-field side (HFS), effectively reducing the poloidal asymmetries as a result of $\mathrm{W}$ accumulation at the LFS by plasma rotation. Poloidal asymmetries have been identified to enhance neoclassical transport and to be the main mechanism for central tungsten accumulation $[29,32]$.

In this paper the analysis has been restricted to hybrid discharges of the campaign with different ICRF resonance positions. Two discharges suffered central impurity accumulation which was accompanied by MHD activity. MHD activity has been resported as the cause of triggering impurity accumulation in hybrid discharges in Ref. [34]. Here, an analysis of the differences of ICRF heating profiles for a scan in the resonance position is performed and temperature screening factors associated with fast minority ions are calculated taking into account orbit width effects. However, modelling of $\mathrm{W}$ transport has not been carried out as it is out of the scope of the present paper.

\section{Numerical methods}

The hybrid discharges simulated in this paper have two competing ion damping mechanisms, the fundamental $H$ resonance and the $2^{\text {nd }}$ harmonic $\mathrm{D}$ resonance $\left(\omega \approx \omega_{H}=\right.$ $\left.2 \omega_{D}\right)$. An accurate description of the velocity distribution of both resonant species is necessary, particularly for higher harmonics $n \geq 2$, where the absorption strength is weak 
at low perpendicular velocities but increases with higher temperatures until a maximum is reached, typically in the $\mathrm{MeV}$ range [36]. For this reason, taking into account the $\mathrm{D}$ beams in the velocity distribution function to solve the wave absorption becomes crucial in this scenario. In order to do so, we use the ICRF code PION [23] which solves the power absorption and the velocity distribution function in a self-consistent way [37]. In general, the input data necessary to run the PION code is obtained from the JET experimental database, which provides the necessary information to simulate each discharge. Data read by PION is stored for different time points and normalized squared root poloidal flux surfaces of a single discharge, therefore, simulations show the evolution in time of absorption profiles and distribution of velocities of resonant ions for the discharge.

The beam source terms are calculated using the beam code PENCIL [24]. These source terms are then included in the Fokker-Planck distribution function of the PION code for each time point and flux surface [11]. For a given flux surface and injection energy, the NBI source is assumed to be Gaussian in velocity for numerical reasons, with the peak centred at the injection energy corrected for bulk plasma rotation effects. The PION code does not calculate the radial transport (except that due to finite orbit widths), hence, in order to keep the consistence between the measured plasma and specially the resonating ion densities with the simulated ones, an ad-hoc procedure is implemented. If the difference of particle density on a flux surface at the beginning and at the end of one time step is higher than the increase in particle density due to NBI, a Maxwellian portion is added to the distribution function in order to compensate. Conversely, if the increase in the number of particles due to NBI on a flux surface during one time step is higher than the measured density, particle losses are introduced to maintain the consistence. In this case, particles are removed from the thermal distribution if losses do not exceed $25 \%$ of the total number of particles at the beginning of the time step, however, if losses exceed this threshold, the removal of particles is applied to the whole distribution function.

\section{General parameters of the hybrid scenario studied}

A summary of the main parameters for the studied discharges is shown in table 1 . The hybrid discharges presented in the following sections have the same plasma composition, i.e. a small concentration in the range of $1-4 \%$ of $\mathrm{H}$ in a $\mathrm{D}$ plasma. ICRF and NBI heating were present in all discharges and minority heating of $\mathrm{H}$ and $2^{\text {nd }} \mathrm{D}$ harmonic heating was the ICRF scheme used. All discharges are explained in each section and are focused on investigating certain key ICRF heating aspects that have an impact on the fusion neutron rate. 
Table 1: JET main parameters for studied hybrid scenario discharges.

\begin{tabular}{lr}
\hline Parameter & \\
\hline Major radius $R_{0}(\mathrm{~m})$ & 2.96 \\
Minor radius $a(\mathrm{~m})$ & $1.25-2.10$ \\
Toroidal magnetic field $B(\mathrm{~T})$ & 2.8 \\
Plasma current Ip $(\mathrm{MA})$ & 2.2 \\
Normalised Beta $\beta_{N}$ & $1.9-2.7$ \\
NBI Power $\mathrm{P}_{\mathrm{NBI}}(M W)$ & $20-26$ \\
ICRF Power $\mathrm{P}_{\mathrm{ICRF}}(M W)$ & $4-5$ \\
Central ion temperature $T_{i 0}(\mathrm{keV})$ & $8-12$ \\
Central electron temperature $T_{e 0}(\mathrm{keV})$ & $6-8.5$ \\
Electron density $n_{e}\left(10^{19} \mathrm{~m}^{-3}\right)$ & $6-7.5$ \\
\hline
\end{tabular}

\section{Effect of resonance position on ICRF impurity control}

The analysis of impurity accumulation is complex as many mechanisms are involved in the transport of high-Z impurities. One of such mechanisms is the temperature screening provided by fast ions generated through ICRF heating. Here, the impurity screening associated to $\mathrm{H}$ minority and the ICRF heating profiles are computed using PION for discharges where resonance position was varied.

The ICRF resonance position was varied in a total of five hybrid discharges in order to assess its impact on the ICRF impurity control. As $R_{\text {res }} \propto B_{T}$, the magnetic field $B_{T}$ was modified accordingly so as to place the ICRF resonance at different major radii ranging from $R_{\text {res }}=2.75 \mathrm{~m}$ (high-field-side) to $R_{\text {res }}=3.2 \mathrm{~m}$ (low-field-side) while the magnetic axis was located at $R_{0}=3.0 \mathrm{~m}$. A total of $30 \mathrm{MW}$ of combined external heating power was used [25], consisting of $25 \mathrm{MW}$ of NBI and $5 \mathrm{MW}$ of ICRF at a frequency of $42 \mathrm{MHz}$. An overview of these discharges is shown in figure 2. In order to keep a similar central safety factor among these discharges, the starting time of the external power heating, i.e. ICRF and NBI heating, was delayed $0.25 \mathrm{~s}$ for each $B_{T}$ change of $0.1 \mathrm{~T}$. Figure 2 shows that those discharges with a resonance $\left|R_{\text {res }}-R_{0}\right|>15$ $\mathrm{cm}$ suffered from MHD activity and impurity accumulation in these plasma conditions. The MHD modes $(\mathrm{m} / \mathrm{n})$ that were present in the HFS discharge 92311 were $3 / 2$ and $4 / 3$ and assuming the EFIT safety factor profile they were located at $\mathrm{s}=0.28$ and $\mathrm{s}$ $=0.03$, respectively. For the LFS discharge 92313 the modes were $5 / 3$ and $4 / 3$ located at $\mathrm{s}=0.34$ and $\mathrm{s}=0.03$, respectively. We have selected three discharges as shown in table 2 for detailed analysis. We refer to them from now on by their ICRF resonance position: HFS, central and LFS.

Figure 3 shows the electron temperature and density for the HFS, central and LFS discharges. During the MHD activity that occurred in the HFS and LFS discharges the central electron density became peaked while the central electron temperature decreased 


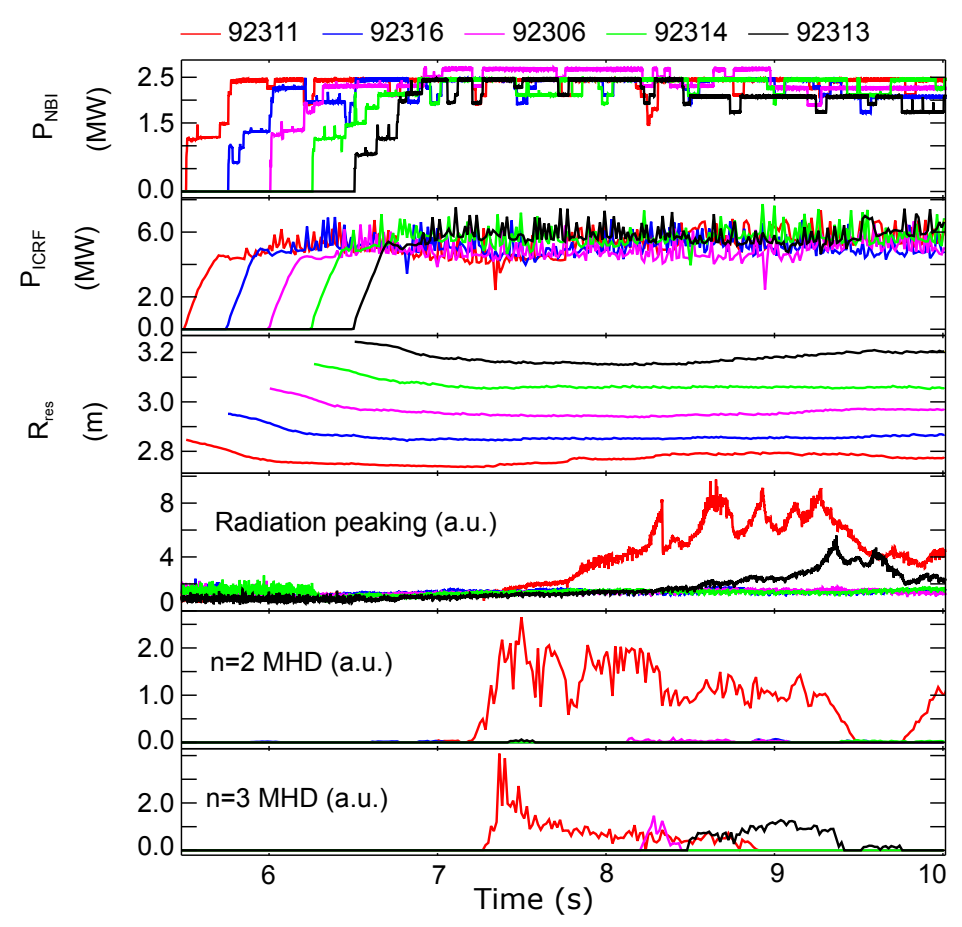

Figure 2: Overview of five hybrid discharges with combined NBI+ICRF heating at different toroidal magnetic fields to vary the ICRF resonance location: 2.6, 2.7, 2.8, 2.9 and $3.0 \mathrm{~T}$ in discharge 92311, 92316, 92306, 92314 and 92313, respectively. The start time of external heating was modified to match the central safety factor at the heating onset.

Table 2: ICRF resonance position for discharges 92311, 92313 and 92314.

\begin{tabular}{cccc}
\hline & 92311 & 92314 & 92313 \\
\hline ICRF resonance pos. & HFS & Central & LFS \\
\hline
\end{tabular}

and became flat in the centre, both effects enhance inward convection (4) which resulted in central W accumulation with a concentration higher than $10^{-4}$. Regarding fusion performance, figure 4 shows that only the discharge with a central resonance avoided impurity accumulation and maintained steady values for $\beta_{N}$, neutron production rate and electron temperature.

The modelled heating profiles (figure 5) show differences as the resonance changes from the HFS to the LFS. The evaluation of the heating profile provides valuable information regarding where the wave energy is being deposited and how strong fast ion pressure can be expected. Typically, central power deposition is preferable (s $<0.3$ ) in order to obtain peaked plasma temperature profiles. PION takes into account orbit width effects which tend to broaden the orbits of the fastest ions and, therefore, collisional electron heating profile broadens with respect to bulk ion heating. Figure 5 shows that ion heating is marginally central for the HFS discharge as bulk ion heating 

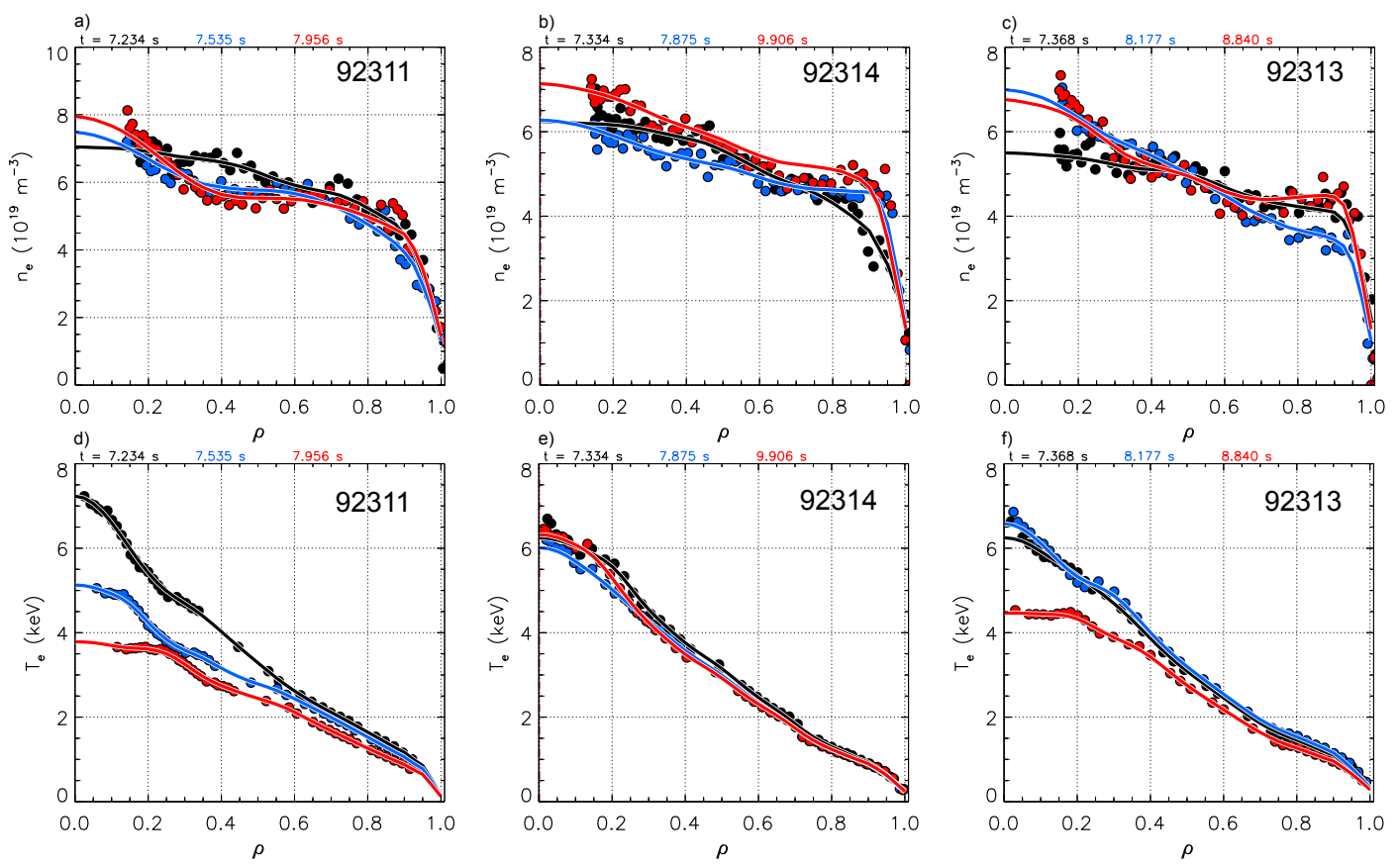

Figure 3: Electron density and temperature as measured by the high resolution Thomson scattering (HRTS) and electron cyclotron emission (ECE) diagnostics, respectively, for HFS discharge 92311 (a) and d)), central discharge 92314 (b) and e)) and LFS discharge 92313 (c) and $f)$ ) at different time points. The flux coordinate $\rho$ is the normalised toroidal flux coordinate. Appearance of MHD and impurity accumulation is around 7.5 $s$ and 8.5 s for discharges 92311 and 92313, respectively.

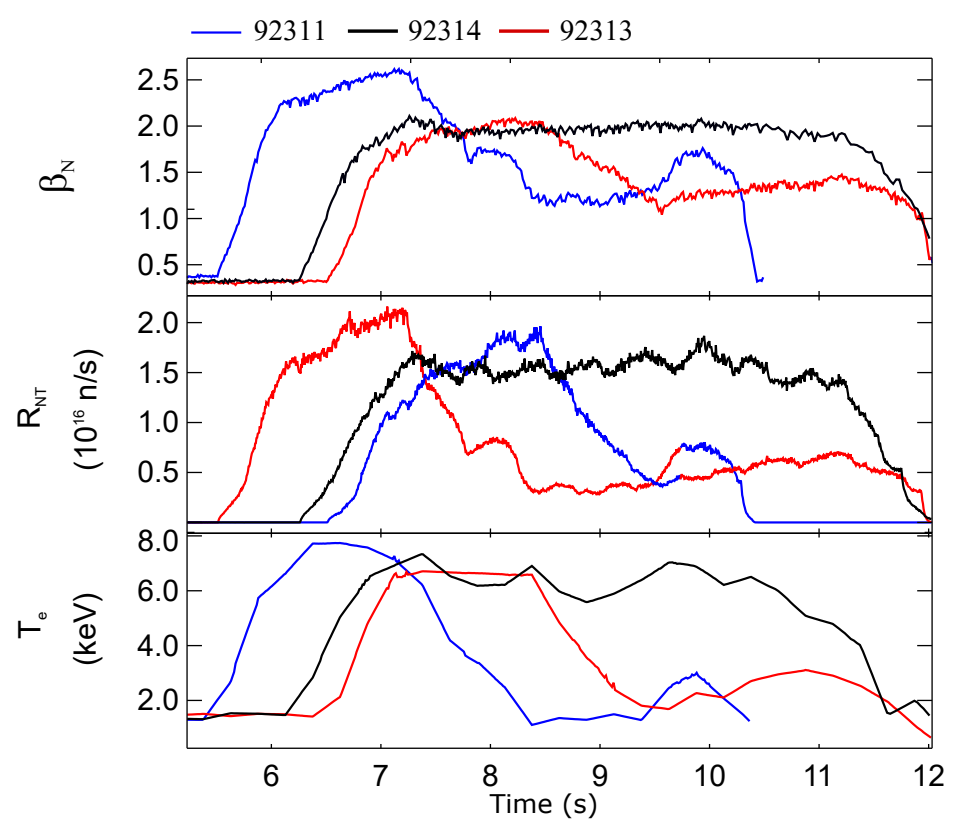

Figure 4: Measured values for $\beta_{N}$, neutron production rate and electron temperature for discharges 92311 (HFS), 92314 (central) and 92313 (LFS). 


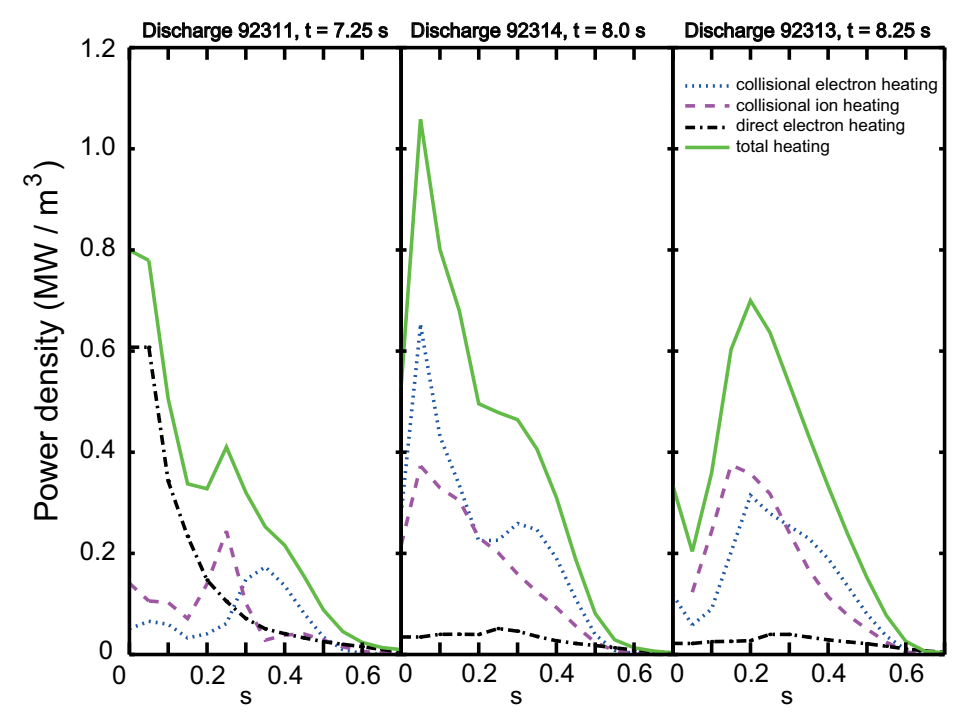

Figure 5: Power density profiles for collisional ion heating, collisional electron heating, direct electron heating and total plasma heating due to ICRF waves as given by PION for discharges 92311, 92314 and 92313 with a HFS, central and LFS ICRF resonance, respectively. Here, $s$ is the square-root of the normalised poloidal flux and the profiles are shown after $1.75 \mathrm{~s}$ from the start of the main heating.

peaks at $\mathrm{s}=0.25$ and collisional electron heating at $\mathrm{s}=0.35$, for the central discharge the power deposition peaks at the centre around $\mathrm{s}=0.1$ and for the LFS around $\mathrm{s}=0.2$. PION predicts strong central direct electron damping for HFS resonance discharge and becomes almost negligible as the resonance is placed towards the LFS. In fact, the highest electron temperature was obtained in the HFS discharge until the appearance of MHD and impurity accumulation (figure 4). The power density of bulk ion heating and collisional electron heating are higher for central and LFS discharges. Collisional electron heating is the dominant heating mechanism in the central discharge which is beneficial to avoid central impurity accumulation as higher fast ion pressure and more peaked plasma temperature profiles are expected. The LFS discharge shows a similar bulk ion heating and collisional electron heating with the total heating power peak located off-axis around $\mathrm{s}=0.2$ while in the other two cases the total heating power peak is located in the centre.

The heating profiles predicted by PION have a direct impact on the calculated fast ion effective temperature. Not only on the average energy of the fast ion population but also on the position where the effective temperature is maximum which is important in order to compute the temperature gradients associated with the impurity screening (4). Figure 6 shows the effective temperature of fast minority for the HFS, central and LFS discharges. The effective temperature has been computed using $T_{\mathrm{f}}=\frac{2 E_{\mathrm{f}}}{3 n_{\mathrm{f}}}$ where $n_{\mathrm{f}}$ and $E_{\mathrm{f}}$ refer to the fast $\mathrm{H}$ minority density and energy density, respectively. Notice that the effective temperature broadens together with the collisional electron heating profiles due to orbit width effects. We have evaluated the temperature screening by fast minority 


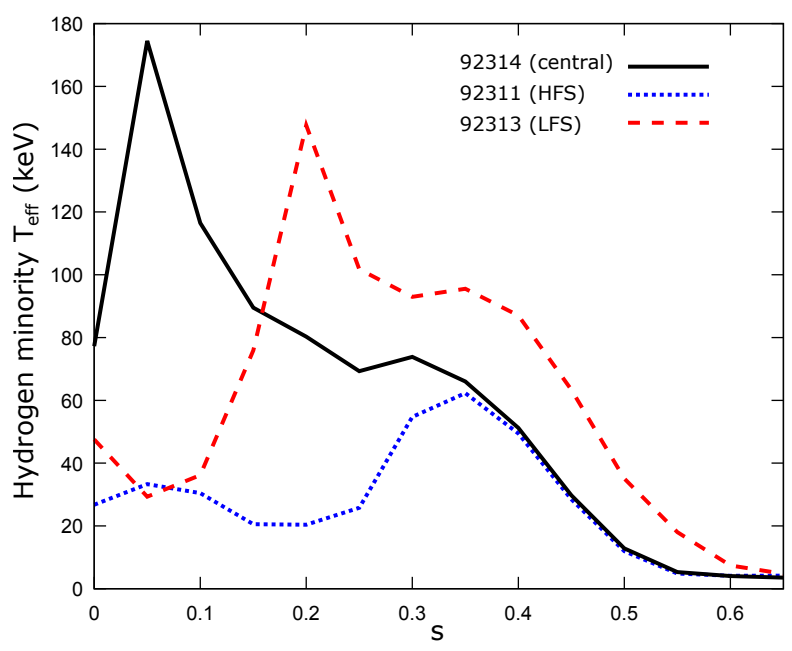

Figure 6: Effective temperature of hydrogen minority ions for discharges 92311 (dotted), 92313 (dashed) and 92314 (solid).

ions in comparison to the relative temperature screening associated to thermal $\mathrm{D}$ as $\Gamma_{\mathrm{f}, \mathrm{H}} / \Gamma_{\mathrm{th}, \mathrm{D}}=n_{\mathrm{f}, \mathrm{H}} T_{\mathrm{th}, \mathrm{D}}^{1 / 2} L_{\mathrm{th}, \mathrm{D}} /\left(n_{\mathrm{th}, \mathrm{D}} T_{\mathrm{f}, \mathrm{H}}^{1 / 2} L_{\mathrm{f}, \mathrm{H}}\right)$. The results of our analysis are shown in table 3. These results show that under these conditions impurity screening from fast

Table 3: Computed temperature screening factor of $\mathrm{H}$ minority normalised to temperature screening factor provided by thermal $\mathrm{D}\left(\Gamma_{\mathrm{f}, \mathrm{H}} / \Gamma_{\mathrm{th}, \mathrm{D}}\right)$ for discharges 92311 (HFS), 92314 (central) and 92313 (LFS).

\begin{tabular}{lccc}
\hline $\mathrm{s}$ & HFS (\%) & Central (\%) & LFS (\%) \\
\hline 0.05 & 0.8 & 2.0 & 1.3 \\
0.10 & 0.2 & 1.4 & -1.2 \\
0.15 & 2.0 & 1.0 & -2.7 \\
0.20 & 0.8 & 1.3 & 2.6 \\
0.25 & -4.0 & 0.0 & -0.1 \\
\hline
\end{tabular}

minority ions is of the order of few percent with respect to that from thermal $\mathrm{D}$. The central discharge shows the highest values for temperature screening in the centre. The HFS discharge has the lowest values of temperature screening except at $\mathrm{s}=0.15$ and a negative value at $\mathrm{s}=0.25$ as a result of a positive temperature gradient which enhances inward flux. In a similar way the LFS discharge has negative values at $\mathrm{s}=0.10$ and $\mathrm{s}$ $=0.15$. These modelling results show that central ICRF heating is beneficial to avoid impurity accumulation as in other studies where it has been studied in detail $[17,18,20]$. However, it is difficult to draw firm conclusions regarding the relevance of fast minority ions among other mechanisms in these conditions as the experimental data show MHD activity and radiation peaking at the same time. For the rest of the hybrid discharges, central ICRF resonance was used. 


\section{Effect of hydrogen concentration on plasma performance}

One of the goals was to assess the impact of the $\mathrm{H}$ concentration on the ICRF heating and optimisation of fusion performance. It is known that the $\mathrm{H}$ minority concentration plays a key role in the ICRF power partitioning (section 2.1) between $\mathrm{H}$ and majority $\mathrm{D}$ and beam-injected $\mathrm{D}$ ions which has an impact in the bulk ion heating and the fusion yield enhancement [11]. A set of discharges with different $\mathrm{H}$ concentration was

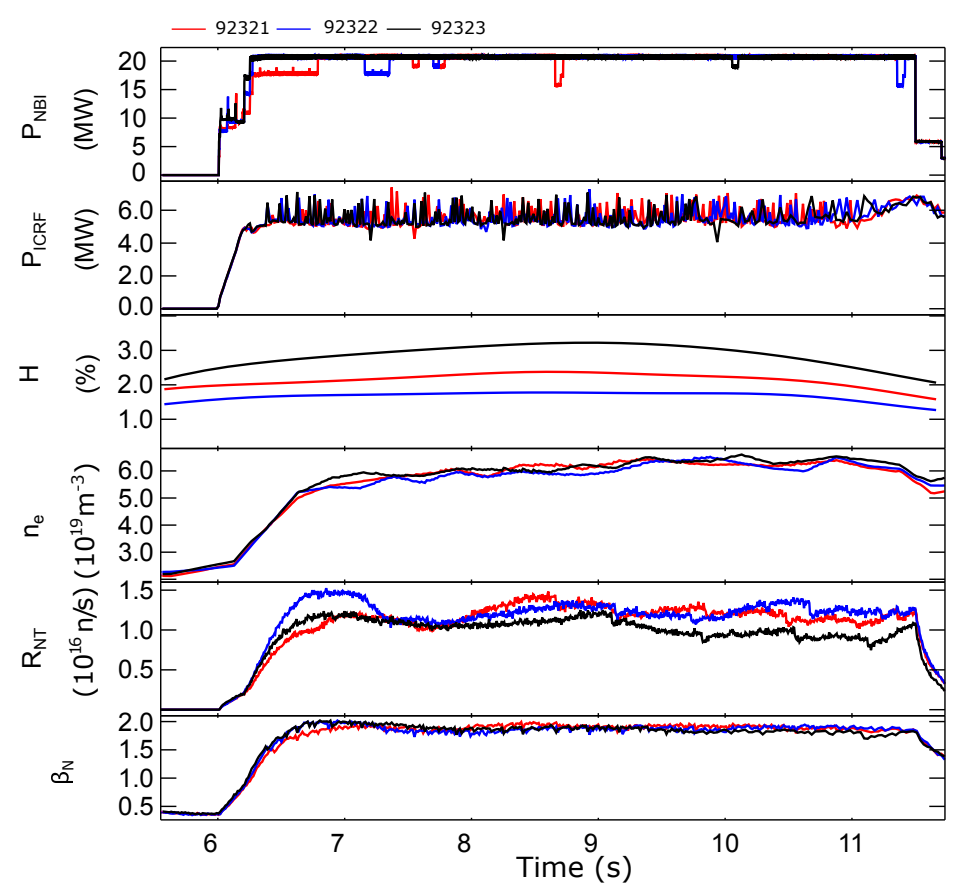

Figure 7: Overview of three hybrid discharges with three different $H$ concentrations. Different neutron rates are obtained for similar plasma parameters and power input as a consequence of slight variations in the $H$ concentration. The $H$ concentration data has been smoothed for this plot.

implemented (figure 7). The same plasma composition and plasma parameters were preserved in these discharges only changing the $\mathrm{H}$ concentration, see table 4 [26]. Here, we quote the $\mathrm{H}$ concentration $n_{H} /\left(n_{H}+n_{D}\right)$ as deduced from the ratio of the $D_{\alpha}$ and $H_{\alpha}$ light collected along lines of sight through the plasma. Penning gauge spectroscopy in the divertor gave somewhat higher $n_{H} /\left(n_{H}+n_{D}\right)$ of $3-4 \%$. According to our modelling, however, the experimental results are more consistent with the values deduced from the $D_{\alpha}$ and $H_{\alpha}$ light (figure 8). The main results in the experimental observations (figure 7) are the differences in the fusion yield for the three discharges. In the early stage of the heating phase up to $t=8 \mathrm{~s}$ discharge with the lowest $H$ puff shows a faster rise in the neutron yield. A lower $\mathrm{H}$ concentration leads to a larger $\mathrm{D}$ damping of the wave energy (figure 9), accelerating them to higher energies with respect to the other discharges (figure 10). As the plasma density grows, the difference in the neutron rate is reduced. From $t=8 \mathrm{~s}$ onwards the fusion yield of the discharge with the lowest $\mathrm{H}$ concentration 
is in average $\sim 20 \%$ higher than in the discharge with the highest $\mathrm{H}$ concentration, which we interpret as being due to a stronger ICRF-accelerated fast deuterium tail.

Table 4: The H concentration for discharges in the $\mathrm{H}$ concentration scan.

$$
\begin{array}{c|ccc} 
& 92321 & 92322 & 92323 \\
\hline n_{H} /\left(n_{H}+n_{D}\right)(\%) & \sim 2.0 & \sim 1.5 & \sim 3.0
\end{array}
$$

The experimental results are in line with our modelling results. Figure 8 shows the simulated neutron rates for the three discharges which are consistent with the measured neutron rates [25]. The difference between the discharge with the lowest $\mathrm{H}$ concentration and the discharge with the highest concentration is about $0-25 \%$ which is in good agreement with the experimental results. The difference vary as the $\mathrm{H}$ concentration is not constant throughout the discharge, the $\mathrm{H}(\%)$ showed in figure 7 has been smoothed for a better visualisation. The ICRF wave was tuned for a central $\omega=\omega_{c H}=2 \omega_{c D}$

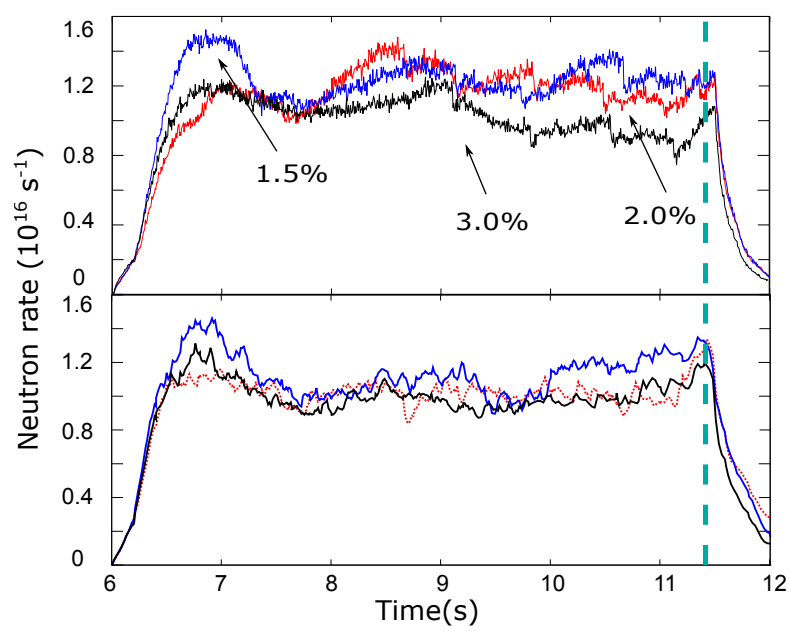

Figure 8: Comparison of experimental neutron emission rate (top) for discharges 92321, 92322 and 92323 with a $H$ concentration of about 2\%, 1.5\% and 3\%, respectively. Modelled neutron emission rate of the $H$ scan discharges (bottom). The vertical dashed line marks the time point at which the ICRF heating profiles are shown.

resonance for the three discharges. The variation of $\mathrm{H}$ concentration has a direct impact on the power partition between the $\mathrm{H}$ and $\mathrm{D}$ ions, this is shown in figure 9 at $\mathrm{t}=11.5$ $\mathrm{s}$ during the main heating phase. In first order, the ratio of $\mathrm{H}$ to $\mathrm{D}$ damping scales roughly as $n_{H} /\left(n_{H}+n_{D}\right)$, as expected. The $\mathrm{H}$ concentration for these discharges is low, however, notice that small differences in the $\mathrm{H}$ concentration play a relevant role in this scenario as they have an impact in the way plasma damps the ICRF wave energy and consequently in the plasma performance. This is clearly evident in figure 10 where a lower $\mathrm{H}$ concentration leads to a stronger highly energetic $\mathrm{D}$ tail, further enhancing fusion performance as the second harmonic D damping has an advantageous effect on the fusion yield. The $\mathrm{D}$ velocity distribution function shows the same trend in the 
PION modelling and in the measurements with the neutron time-of-flight spectrometer TOFOR [38,39], we have not attempted the full comparison in absolute units as this very detailed comparison is beyond the purposes of the present paper, however, comparisons have been carried out in [40]. Notice that the cross section for DD fusion reactions peak at the $\mathrm{MeV}$ range and, therefore, strengthening the ICRF-accelerated deuterium tail enhances the fusion neutron rate for this particular case. As it is shown in figure 9,

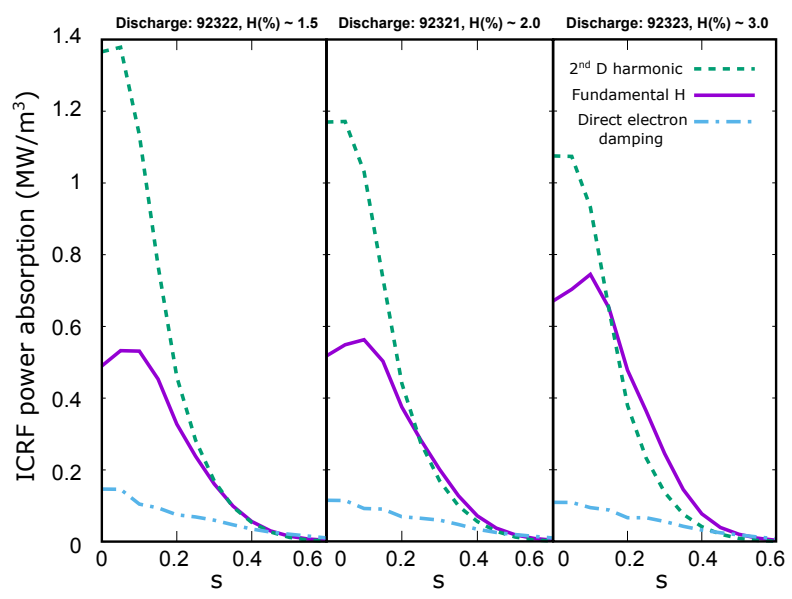

Figure 9: ICRF power absorption profile for fundamental $H$ (solid), $2^{\text {nd }} D$ harmonic resonance (dashed) and direct electron damping (dashed-dotted) at $t=11.5 \mathrm{~s}$.

after the deuterium beams have been injected, $2^{\text {nd }} \mathrm{D}$ harmonic resonance becomes the main damping mechanism at the plasma centre once the plasma core gets hotter, while fundamental $\mathrm{H}$ resonance dominates for low plasma densities and temperatures that take place during the ramp up. Small to no differences are predicted by PION with regards to direct electron damping, therefore, varying the $\mathrm{H}$ concentration in a small range has no impact in the total fraction of energy absorbed by ions and electrons but only in the competing damping mechanisms by ion species.

Figure 11 shows the time evolution of fundamental $\mathrm{H}$ absorption, $2^{\text {nd }} \mathrm{D}$ harmonic absorption and direct electron damping for discharges with the lowest and highest $\mathrm{H}$ concentration. Similar behaviour is observed in the way damping mechanisms evolve, i.e. fundamental $\mathrm{H}$ damping dominates during the ramp up while it fastly decays once the $\mathrm{D}$ beams are injected as they produce a rapid increase of the $2^{\text {nd }} \mathrm{D}$ harmonic damping strength. As the $\mathrm{H}$ concentration decreased from $3 \%$ to $1.5 \%$, D damping increased from $35 \%$ to $50 \%$ and $\mathrm{H}$ damping decreased from $50 \%$ to $35 \%$ in average, while direct electron damping stayed roughly the same, i.e. $15 \%$ of the total ICRF power.

\section{High-performance hybrid discharge}

From the point of view of fusion neutron rate, there was the aim to improve the previous record of $2.3 \cdot 10^{16} \mathrm{n} / \mathrm{s}$ from hybrid discharge 86614 . The neutron rate record was achieved in several discharges and here, we study the combined effect of ICRF and NBI heating 

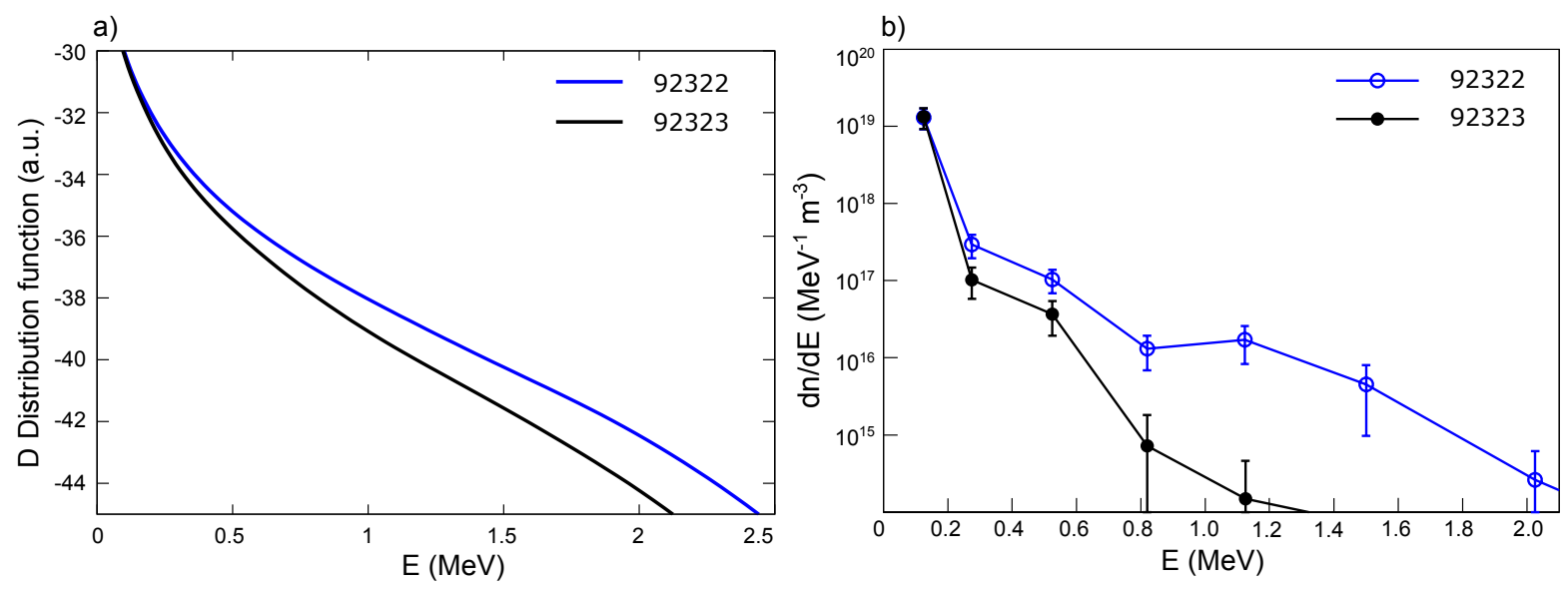

Figure 10: Energy distribution functions of deuterium in logarithmic scale for discharges 92322 (black) and 92323 (blue) with a $H$ concentration of about $1.5 \%$ and 3\%, respectively. a) Deuterium distribution function as calculated by PION close to the resonance position and b) as deduced from measurements with the neutron time-of-flight spectrometer TOFOR.

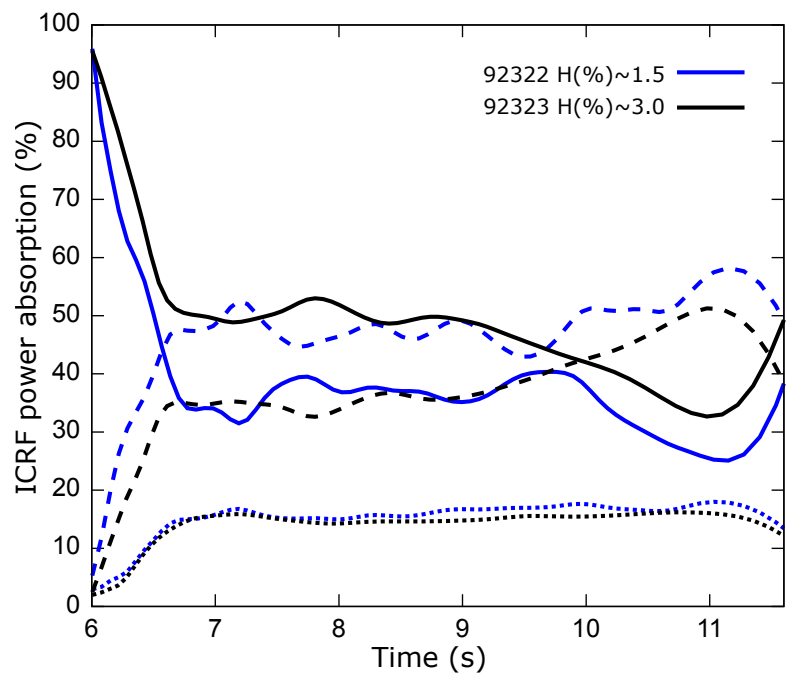

Figure 11: Damping fractions of the total ICRF power with fundamental hydrogen absorption (solid), $2^{\text {nd }}$ deuterium harmonic absorption (dashed) and direct electron damping (dotted) for discharges 92322 (blue) and 92323 (black).

in one of the best shots with $B_{T}=2.8 \mathrm{~T}$ and $I_{p}=2.2 \mathrm{MA}$, discharge 92398. Similarly to other hybrid discharges, $5 \mathrm{MW}$ of ICRF power was tuned to a central fundamental $\mathrm{H}$ and second $\mathrm{D}$ harmonic resonance $\left(\omega=\omega_{H}=2 \omega_{D}\right)$ using $42.5 \mathrm{MHz}$. Together with deuterium NBI power of $26 \mathrm{MW}$, the total external heating power was $31 \mathrm{MW}$. An overview of the high performing hybrid discharge 92398 is presented in figure 12 . The neutron rate and normalised plasma beta increased to their steady-state values of $2.7 \cdot 10^{16} \mathrm{~s}^{-1}$ and 2.7 , respectively, when stationary high-performance plasma was obtained. 


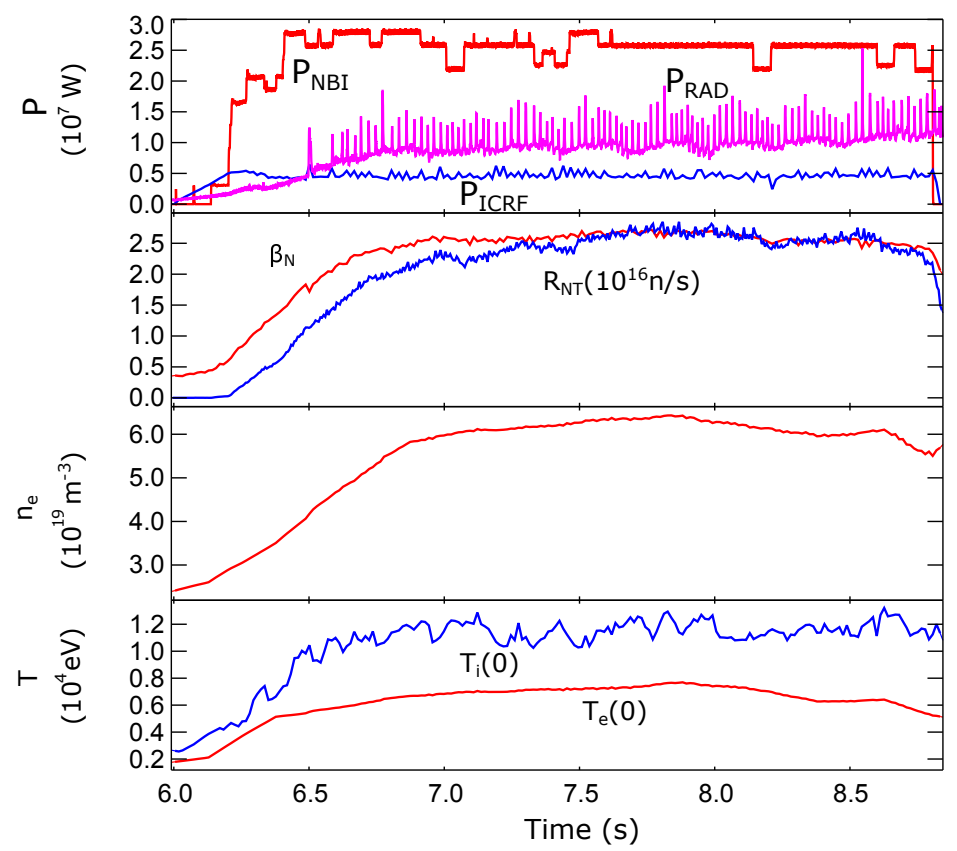

Figure 12: Overview of the high performing hybrid dicharge 92398. A factor $R=$ $T_{i} / T_{e}$ has been applied to the $T_{i}$ as measured by the X-ray crystal spectroscopy (XCS) diagnostic.

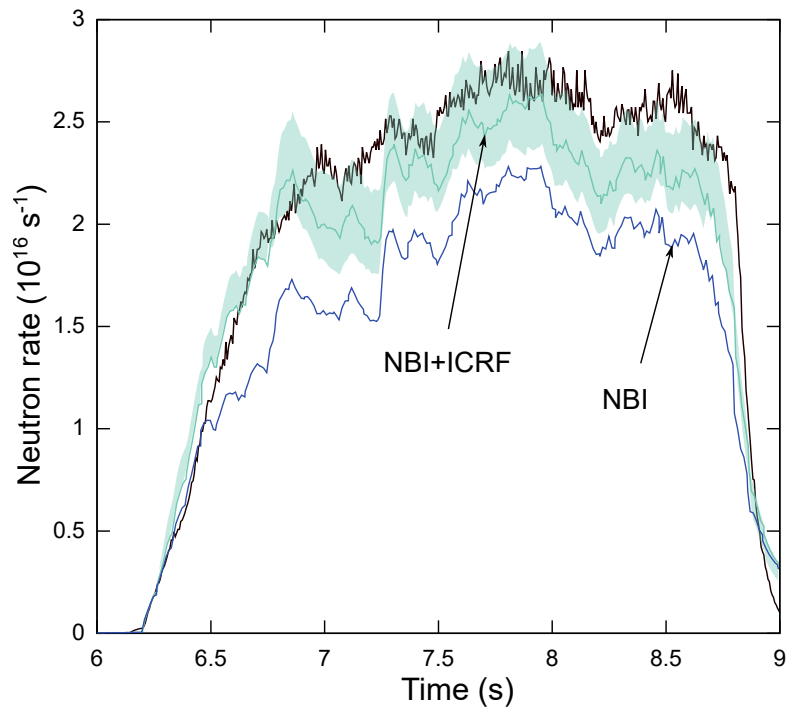

Figure 13: Neutron production rate modelling with error bars (shaded area) of discharge 92398 with ICRF and without ICRF.

The modelling has been performed with the PION code and shows excellent agreement not only with the measured neutron rate (figure 13) but also with the ICRF enhancement of the neutron rate (figure 14). The experimental ion temperature $T_{i}$ has been computed using the $T_{i} / T_{e}$ ratio as deduced from the X-ray crystal spectroscopy data. This data suggests that the $T_{i} / T_{e}$ ratio is around 1.25-1.6. The $\mathrm{H}$ concentration $n_{H} /\left(n_{H}+n_{D}\right)$ deduced from the ratio of the $\mathrm{D}_{\alpha}$ and $\mathrm{H}_{\alpha}$ light collected along lines of sight 
through the plasma is $\sim 2 \%$ and has been used to model this discharge. The calculated and experimental neutron yield are in good agreement as shown in figure 13. However, several uncertainties associated with the experimental measurements have been taken into account to assess their impact on the neutron yield (shaded area in figure 13). In particular, we have taken into account uncertainties in the impurity content and the ion temperature.

One of the main goals was to evaluate the impact of ICRF enhancement in the fusion yield. In order to do so, the neutron rate has been modelled in two different ways, one which takes into account the full external power and a second one which only models the NBI heating (figure 13). The NBI only case has been modelled with PION with zero antenna power and using the beam source terms from PENCIL. The ICRF enhancement is calculated from the difference between the associated neutron yields. Figure 14 shows a good agreement between the ICRF enhancement as estimated from a spectroscopic analysis of data collected by the neutron time-of-flight spectrometer TOFOR and as predicted by the PION code. The enhancement varies throughout the discharge, starting at a maximum value of around $35 \%$ during the ramp-up phase and reaching a steady value of $15 \%$ during the main heating phase. This variation is the result of an increasing plasma density and temperature, as the plasma becomes hotter the neutron emission from D beams becomes dominant and, therefore, the ICRF enhancement is reduced until a steady value. However, notice that this enhancement does not extrapolate to DT scenario as will be shown in section 8.2 since the fusion cross sections $\sigma_{D T}$ and $\sigma_{D D}$ are different.

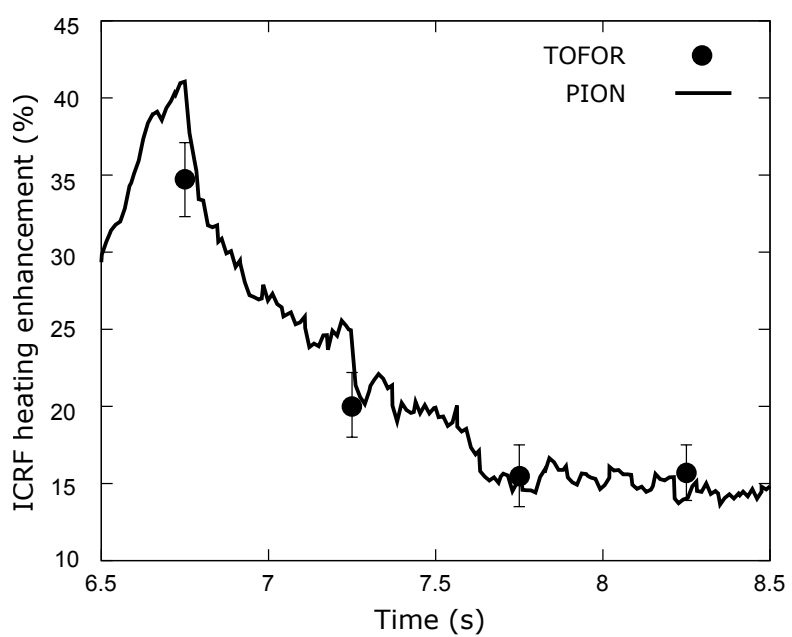

Figure 14: ICRF enhancement of neutron yield for discharge 92398. Comparison of PION (solid line) and experimental results based on TOFOR (black dots) measurements.

In order to maximise the ICRF fusion enhancement in this scenario, it is crucial that $\mathrm{D}$ ions absorb most of the ICRF wave power and the presence of a high bulk ion heating as mentioned in section 2.2. The damping mechanisms competing in this discharge are the same as those described in section 6 and the physics are similar. During the ramp- 
up, fundamental $\mathrm{H}$ absorbs almost all the wave power while $2^{\text {nd }} \mathrm{D}$ harmonic damping becomes dominant during the main heating phase (figure 15 a)). The absorption profile (figure 16) shows a strong absorption of fundamental $\mathrm{H}$ for low plasma temperatures while it becomes lower during the main heating phase where $\mathrm{D}$ absorption becomes dominant as seen in section 6. Bulk ion heating is dominant throughout the whole discharge except for the low temperatures and densities that characterise the ramp-up phase (figure $15 \mathrm{~b}$ )), where fundamental $\mathrm{H}$ strongly dominates generating a fast ion population that slows-down mainly through ion-electron collisions. PION predicts an average fast ion energy of $500 \mathrm{keV}$ for minority $\mathrm{H}$ at $\mathrm{t}=6.25 \mathrm{~s}$ where fundamental $\mathrm{H}$ absorption peaks and 160 and $65 \mathrm{keV}$ for $\mathrm{H}$ and $\mathrm{D}$, respectively, during the main heating phase. The computed critical energies are 115 and $230 \mathrm{keV}$ for $\mathrm{H}$ and $\mathrm{D}$, respectively, during the main heating phase. The fact that bulk ion heating dominates is in line with the measured higher $T_{i}$ than $T_{e}$ for this particular discharge.
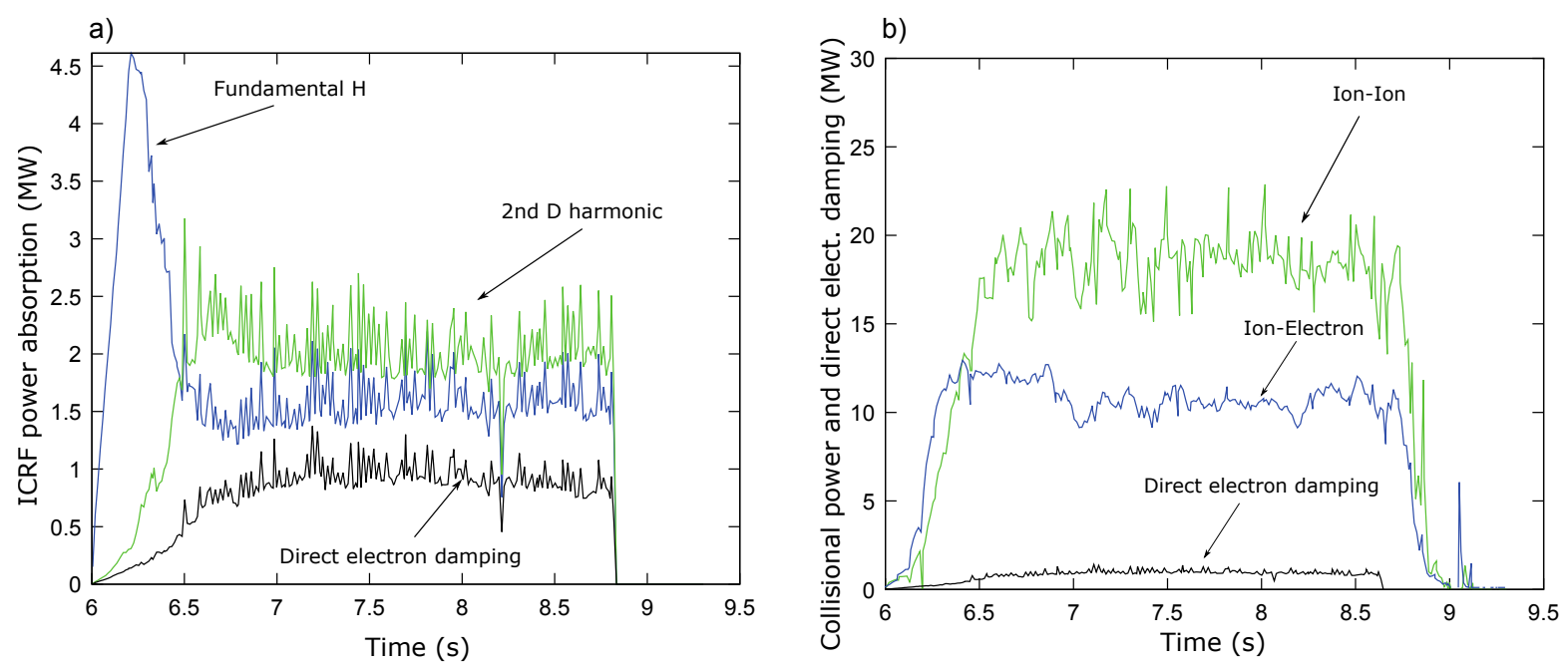

Figure 15: a) ICRF power absorption mechanisms, fundamental $H$, $2^{\text {nd }} D$ harmonic and direct electron damping for discharge 92398. b) Direct electron damping and collisional power transferred to ions and electrons from ion-ion collisions and ionelectron collisions, respectively.

\section{Predictions for DT}

The prediction for a DT discharge is the last step in the analysis of the high-performance hybrid discharges, where the maximum power output and the best strategy to follow is studied. This section tackles two different topics: the DT fusion yield and the maximization of bulk ion heating from the prediction of a high-performance hybrid discharge. 


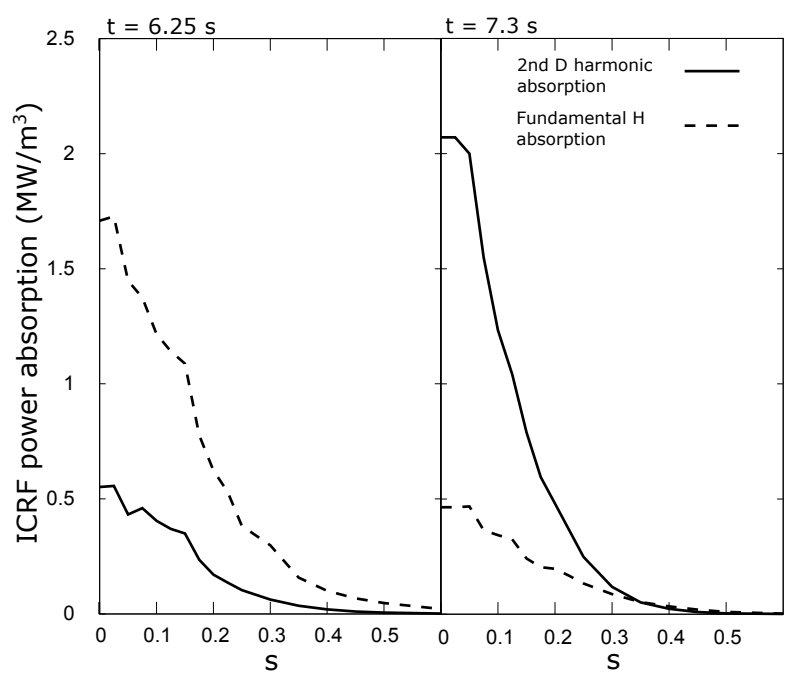

Figure 16: Absorption profile for fundamental $H$ absorption (dashed) and $2^{\text {nd }} D$ harmonic absorption (solid) at $t=6.25 \mathrm{~s}$ and $t=7.3 \mathrm{~s}$.

\subsection{DT fusion yield prediction}

In order to model a 50\%:50\% DT plasma from the high-performance discharge 92398, the plasma composition has been modified by changing half of the bulk D by $\mathrm{T}$ and half of the D beams by $\mathrm{T}$. In this way, the rest of the plasma parameters remain unchanged with respect to those in the experiment. In this new scenario, the ICRF scheme changes as $\mathrm{T}$ becomes resonant together with $\mathrm{H}$ and $\mathrm{D}$. However, as discussed in section (2.1) and showed in equation (2) the damping strength of $3^{\text {rd }} \mathrm{T}$ harmonic is negligible as compared to that of $\mathrm{H}$ or $\mathrm{D}$. In fact, as $\mathrm{D}$ and $\mathrm{T}$ beams are injected, the $2^{\text {nd }} \mathrm{D}$ harmonic becomes the dominant damping mechanism as expected. Nevertheless, while previous sections hinged around the idea that channeling most of the power to D was beneficial for the fusion performance, it does not necessarily apply in the DT scenario (section 2.2). The reason is that fusion DD and DT cross sections peak at different energy regions, while $\mathrm{DD}$ cross section peaks around the $\mathrm{MeV}$ range, $\mathrm{D} \rightarrow \mathrm{T}$ cross section peaks around 120 $\mathrm{keV}$ and decreases rapidly beyond this energy. Therefore, one needs to be careful in the way energy is channeled to D as a strong high energy tail in the distribution function has the potential to decrease the number of fusion reactions. As beams are close to the optimal energy for DT fusion reactions to occur, most of the fusion reactions are due to beam-thermal ion interactions and thus, a lower ICRF enhancement is expected as compared to the DD scenario which is about $5 \%$ in this case. PION predicts an equivalent DT fusion power of about $7 \mathrm{MW}$.

\subsection{Combined NBI + ICRF heating in JET DT plasmas}

Bulk ion heating and ICRF fusion enhancement are relevant quantities in order to evaluate DT plasmas. An increased neutron rate should be obtained by maximising both. The following discussion shows the results obtained with coupled PENCIL and 
PION and studies the dependence of bulk ion heating and ICRF fusion enhancement under a scan on key plasma parameters. A 50\%-50\% DT fuel ion mixture is considered under a scan in plasma temperature and density of the hybrid discharge $86614\left(n_{e}=\right.$ $\left.6.2 \cdot 10^{19} \mathrm{~m}^{-3}, T_{e}=9 \mathrm{keV}\right)$. Ion and electron temperatures are assumed to be equal. The toroidal magnetic field is set to $B_{T}=3.25 \mathrm{~T}$ and the plasma current to $I_{p}=$ 2.7 MA. A total heating power of $40 \mathrm{MW}$ has been simulated consisting of $34 \mathrm{MW}$ of NBI power (17 MW of D beams and $17 \mathrm{MW}$ of $\mathrm{T}$ beams) and $6 \mathrm{MW}$ of ICRF power, in several simulations the ICRF power has been set to zero for comparison purposes. Regarding the ICRF scheme a comparison between $\mathrm{H}$ and ${ }^{3} \mathrm{He}$ as minority species has been performed while the simulated antenna frequency has been set for central heating for all the cases under consideration.

The resonant ion absorption strength for both minority scenarios ranges from $63 \%$ to $87 \%$ and $73 \%$ to $90 \%$ for $\mathrm{H}$ and ${ }^{3} \mathrm{He}$ minority scenarios, respectively (figure 17 ). PION predicts a dependency of the power absorption on both, the plasma density and the plasma temperature. For lower plasma temperatures and densities the power absorption is higher while it decreases for higher values of plasma temperature and density where direct electron damping is stronger as it is $\propto T_{e} n_{e}$. However, ion absorption is dominant over direct electron damping for the whole range under consideration.
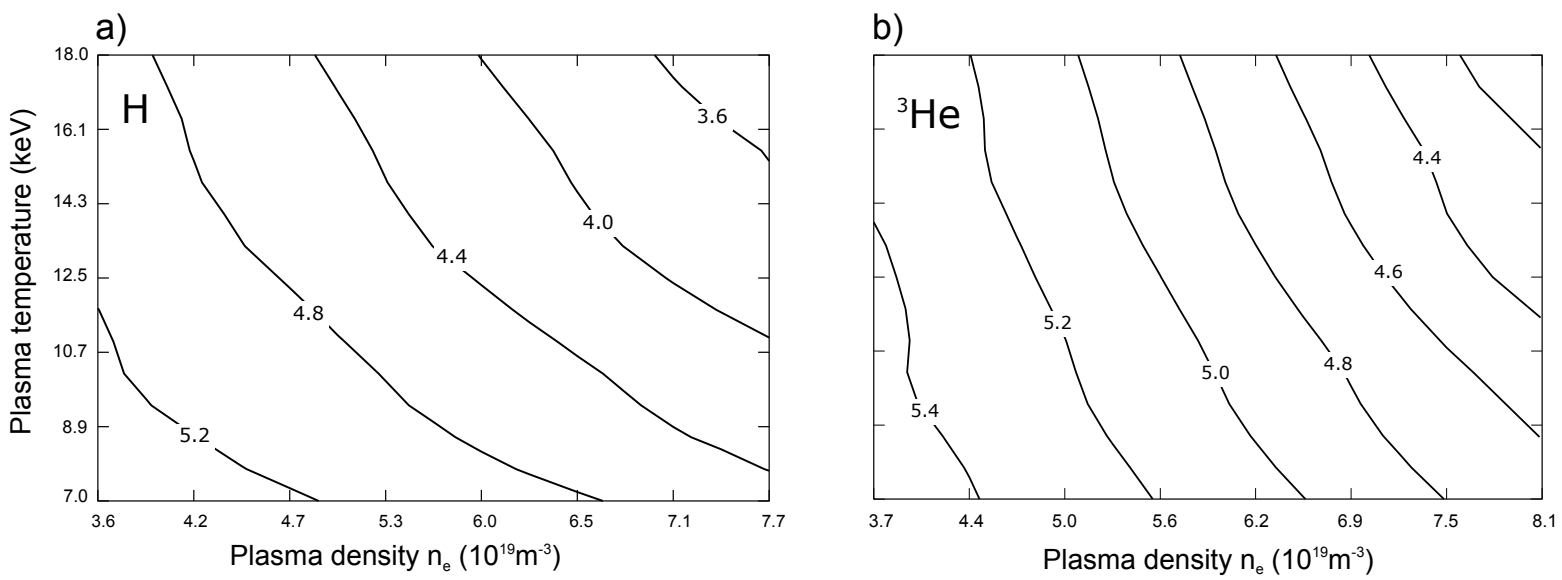

Figure 17: Contour lines of power absorption of resonant ions in $M W$ for a minority concentration of $5 \%$, a) $\mathrm{H}$ minority and b) ${ }^{3} \mathrm{He}$ minority.

Bulk ion heating is shown in figure 18. Although $\mathrm{D}$ and $\mathrm{T}$ beams are used for both minority scenarios, i.e. $\mathrm{H}$ and ${ }^{3} \mathrm{He}$, only resonant species are shown, therefore, only $\mathrm{T}$ beams (17 MW and $\sim 95 \mathrm{keV}$ ) are shown in the ${ }^{3}$ He minority case and only $\mathrm{D}$ beams (17 MW and $\sim 105 \mathrm{keV}$ ) in the $\mathrm{H}$ minority case as they are resonant through the $2^{\text {nd }}$ harmonic resonance. Tritium beams are resonant in the $\mathrm{H}$ minority scenario but the $3^{\text {rd }}$ $\mathrm{T}$ harmonic resonance absorption strength is negligible in front of the $2^{\text {nd }} \mathrm{D}$ harmonic and $\mathrm{H}$ fundamental absorption strength as predicted by PION under the conditions of this scenario.

For all the range under consideration of the pure NBI simulated scenario, between $65-90 \%$ of the D NBI power and $82-100 \%$ of the T NBI power is transferred to ions, this 

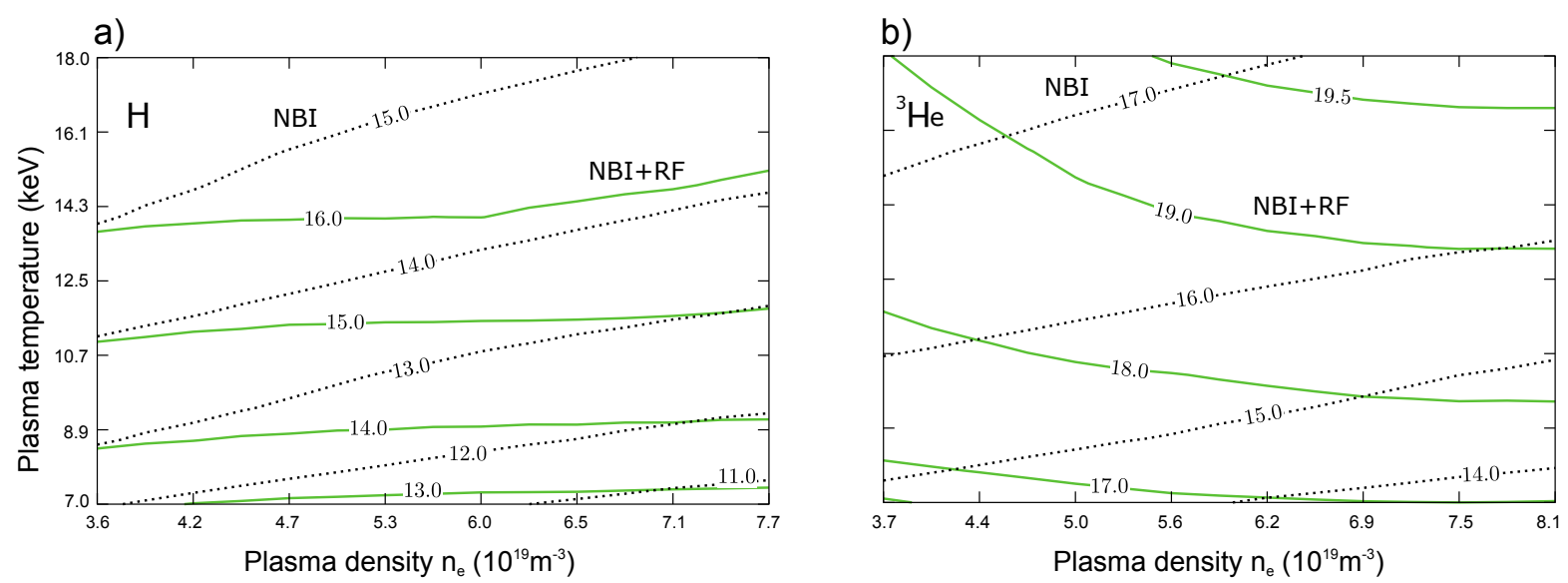

Figure 18: Contour lines of collisional power from resonant ions to thermal ions for NBI and $N B I+R F$ in $M W$ for a minority concentration of $5 \%$, a) $H$ minority and b) ${ }^{3}$ He minority.

difference lies in the higher energy of $\mathrm{D}$ beams and a higher critical energy of $\mathrm{T}$ beams as $E_{\text {crit }} \propto A$ (see (3)). The simulations with NBI+ICRF show a different trend and the differences between both minority scenarios grow. As the critical energy increases with $T_{e}$ the collision power from resonant ions to thermal ions depend mainly on the plasma temperature. However, as compared to the simulated pure NBI scenario, the NBI+RF scenario shows an increase of bulk ion heating with plasma density. The reason why this occurs is that the average energy of fast ions tends to decrease and the direct electron damping becomes relatively stronger for increasing plasma densities. Both effects tend to increase the proportion of fast ion energy transferred to ions by lowering the average fast ion energy (see figure 1). Regarding the differences between both minority scenarios, the ${ }^{3} \mathrm{He}$ minority scenario shows a $15-20 \%$ higher bulk ion heating, mainly due to a higher critical energy of ${ }^{3} \mathrm{He}$ as compared to $\mathrm{H}$ and the lower direct electron damping in the ${ }^{3}$ He minority scenario. A scan in the minority concentration at $T_{e}=9 \mathrm{keV}$ and $n_{e}=6 \cdot 10^{19} \mathrm{~m}^{-3}$ is presented in table 5 . Bulk ion heating increases with the minority concentration for both cases as the average fast ion energy decreases. For higher minority concentrations the absorption tends to become weaker for fundamental heating as the polarization of the wave changes due to high minority concentration in the plasma.

Table 5: Bulk ion heating in MW for a scan in the minority concentration.

\begin{tabular}{c|cccc}
\hline Minority & $1 \%$ & $3 \%$ & $5 \%$ & $7 \%$ \\
\hline $\mathrm{H}$ & 13.5 & 13.7 & 13.9 & 14.1 \\
${ }^{3} \mathrm{He}$ & 16.1 & 17.0 & 17.7 & 18.0 \\
\hline
\end{tabular}

The motivation for modelling the ICRF fusion enhancement of the DT scenario (figure 19) comes from the fact that, in principle, ICRF heating can accelerate deuterons and tritons beyond the optimal DT fusion reaction energy $(\sim 120 \mathrm{keV}$ for fast $\mathrm{D}$ and $\sim 160 \mathrm{keV}$ for fast $\mathrm{T}$ ), which could result in a lower fusion yield. However, figure 19 

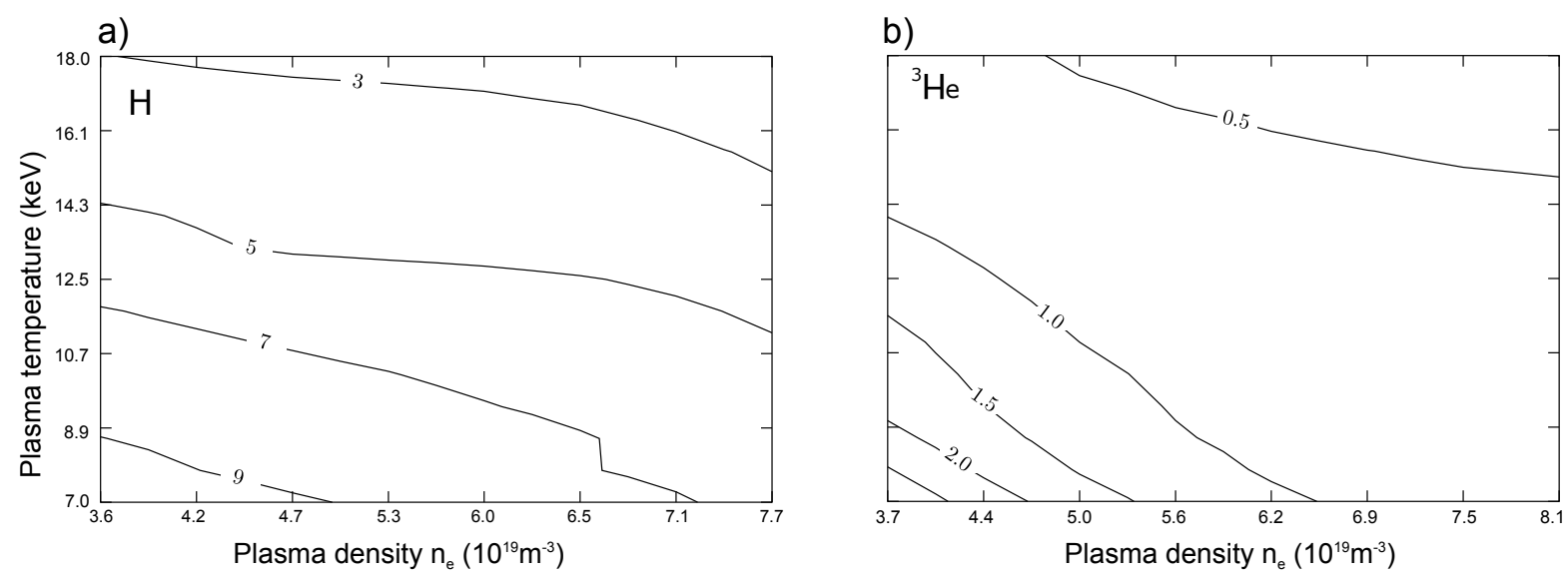

Figure 19: Contour lines of the ICRF fusion enhancement of $D+T$ reaction rate in $\%$ of the total $\frac{R_{N T}(N B I+R F)-R_{N T}(N B I)}{R_{N T}(N B I+R F)}$, a) H minority and b) ${ }^{3}$ He minority.

shows that this is not the case for the parameter range under consideration as the ICRF enhancement is positive in both minority scenarios. The ${ }^{3} \mathrm{He}$ scenario shows a lower ICRF enhancement of the DT fusion reaction rate as the T damping strength is roughly $10 \%$ of the total $\mathrm{D}$ damping strength for the $\mathrm{H}$ minority scenario. Nevertheless, the ICRF fusion enhancement behaves in a similar way for both minority scenarios, it is lowest at regions where thermal fusion reactivity increases, i.e. regions of higher plasma density and temperature. The ICRF enhancement ranges from 2-9\% for $\mathrm{H}$ minority scenario and $0.5-2 \%$ for ${ }^{3} \mathrm{He}$ minority scenario.

\section{Conclusions}

A number of key topics related to the use of ICRF waves in hybrid plasmas have been modelled and analysed in this paper, such as the impact of ICRF resonance location on the impurity accumulation, the importance of $\mathrm{H}$ minority concentration on the ICRF performance and the ICRF properties and neutron yield enhancement of a high-performance hybrid discharge in addition to its DT prediction. It has been shown that heating with ICRF waves centrally has beneficial effects in order to avoid impurity accumulation while heating further away from the centre can cause impurity accumulation under these conditions $\left(\left|R_{\text {res }}-R_{0}\right|>15 \mathrm{~cm}\right)$. In this scenario, channeling the maximum ICRF power to D has an advantageous effect in the fusion yield, ranging from 0 to $25 \%$ by lowering the $\mathrm{H}$ concentration which tends to make $2^{\text {nd }} \mathrm{D}$ harmonic resonance more dominant. Therefore, the modelling of this scenario supports central ICRF heating and low concentration of the minority in order to improve the duration and neutron rate of the discharge. Regarding the ICRF enhancement in the neutron rate, a steady $15 \%$ enhancement was achieved in a high-performance discharge during the main heating phase and 30\% during the ramp-up. However, the enhancement in the DT prediction is diminished, mainly due to the different fusion cross sections of DD and DT fusion reactions. The modelling of the DT prediction shows an improved bulk 
ion heating of the order of $15-20 \%$ for the ${ }^{3} \mathrm{He}$ minority scheme as compared to the $\mathrm{H}$ minority scheme, while ICRF fusion enhancement is predicted to be higher in the $\mathrm{H}$ scheme as $2^{\text {nd }} \mathrm{D}$ harmonic resonance shows a stronger absorption as compared to $2^{\text {nd }} \mathrm{T}$ harmonic resonance in the ${ }^{3} \mathrm{He}$ minority scheme.

\section{Acknowledgements}

One of the authors (D. Gallart) would like to thank Dr. J.C. Wright and Dr. H. Weisen for carefully reading the present work and for their useful comments. D. Gallart would also like to thank Dr. P. Buratti for providing MHD related data. This work has been carried out within the framework of the EUROfusion Consortium and has received funding from the Euratom research and training programme 2014-2018 under grant agreement No 633053. The views and opinions expressed herein do not necessarily reflect those of the European Commission. D. Gallart is grateful to 'la Caixa' for supporting his Phd studies. This work has received funding from the Spanish Ministry of Economy and Competitiveness (MINECO) under grant ENE2015-67371-R.

\section{References}

[1] C.K. Phillips et al., Physics of Plasmas 2 (1995) 2427.

[2] J.R. Wilson et al., Physical Review Letters 75 (1995) 842.

[3] L.-G. Eriksson et al., Nuclear Fusion 39 (1999) 337.

[4] D.F.H. Start et al., Physical Review Letters 80 (1998) 4681.

[5] D.F.H. Start et al., Physical Review Letters 39 (1999) 321.

[6] M.-L. Mayoral et al., Nuclear Fusion 46 (2006) 7 S550.

[7] E.F. Jaeger et al., Physics of Plasmas 15 (2008) 072513.

[8] M.J. Mantsinen et al., Plasma Phys. Control. Fusion 45 (2003) A445-A456.

[9] M.J. Mantsinen et al., Phys. Rev. Lett. 88 (2002) 10105002.

[10] Ye.O. Kazakov et al., Nature Physics 13 (2017) 973.

[11] M.J. Mantsinen et al., Plasma Phys. Control. Fusion 41 (1999) 843.

[12] G.A. Cottrell et al., Nuclear Fusion 39 (1999) 389.

[13] F.G. Rimini et al., Nuclear Fusion 39 (1999) 1591.

[14] R.W. Harvey et al., Nuclear Fusion 26 (1986) 143.

[15] V.B. Krapchev et al., Nuclear Fusion 25 (1985) 4455.

[16] C. Angioni et al., Nuclear Fusion 57 (2017) 056015.

[17] M. Goniche et al., Plasma Phys. Control. Fusion 59 (2017) 055001.

[18] E. Lerche et al., Nuclear Fusion 56 (2016) 3036022.

[19] J. Garcia et al., Plasma Physics and Controlled Fusion 59 (2017) 014023.

[20] T. Casper et al., Nuclear Fusion, 541 (2014) 013005.

[21] C. Gormezano et al., Fusion Science and Technology, 53 (2008) 958.

[22] X. Litaudon et al., Nuclear Fusion, 57 (2017) 102001.

[23] L.-G. Eriksson et al., Nuclear Fusion 33 (1993) 1037.

[24] P.M. Stubberfield et al. Multiple Pencil Beam, JET-DPA(06)/87, 1987.

[25] M.J. Mantsinen et al., European Journal of Physics 157 (2017) 03032.

[26] M.J. Mantsinen et al., Europhysics Conference Abstracts vol. 41F (2017) O3.110.

[27] D. Gallart et al., European Journal of Physics 157 (2017) 03015.

[28] D. Gallart et al., Europhysics Conference Abstracts vol. 40A (2017) P2.003.

[29] F.J. Casson et al. Plasma Phys. Control. Fusion 57 (2015) 014031. 
[30] C. Angioni and P. Helander Plasma Phys. Control. Fusion 56 (2014) 124001.

[31] M.Valisa et al. Nucl. Fusion 51 (2011) 033002.

[32] M.L. Reinke et al. Plasma Phys. Control. Fusion 54 (2012) 045004.

[33] M. Goniche et al. Europhysics Conference Abstracts vol. 38F (2014) 04.129.

[34] T.C. Hender et al. Nuclear Fusion 56 (2016) 066002.

[35] T.H. Stix, Plasma Physics 14 (1972) 367.

[36] L.-G. Eriksson et al. Physics of Plasmas 1 (1994) 308.

[37] L.-G. Eriksson et al. Physica Scripta 55 (1995) 70.

[38] M. Gatu Johnson et al., Nucl. Instrum. Methods A, 591 (2008) 417.

[39] C. Hellesen et al., Nuclear Fusion 53 (2013) 113009.

[40] C. Hellesen et al., Nuclear Fusion 58 (2018) 056021. 